\title{
Investigation of an Anomaly Observed in Impedance Eduction Techniques
}

\author{
W. R. Watson, M. G. Jones, and T. L. Parrott ${ }^{\ddagger}$ \\ NASA Langley Research Center, Hampton, Virginia 23681-2199, USA
}

\begin{abstract}
An intensive investigation into the cause of anomalous behavior commonly observed in impedance eduction techniques is performed. The investigation consists of grid refinement studies, detailed evaluation of results at and near anti-resonance frequencies, comparisons of different model results with synthesized and measured data, assessment of optimization techniques, and evaluation of boundary condition effects. Results show that the root cause of the anomalous behavior is the sensitivity of the educed impedance to small errors in the measured termination resistance at frequencies near anti-resonance or cut-on of a higher-order mode. Evidence is presented to show that the common usage of an anechoic, plane wave termination boundary condition in ducts where the "true" termination is reflective may act as a trigger for these anomalies. Replacing the exit impedance boundary condition by an exit pressure condition is shown to reduce the anomalous results.
\end{abstract}

\section{Nomenclature}

$[A],\{F\} \quad$ finite element system matrix, vector of source effects

$A_{n}, \alpha_{n} \quad$ complex mode coefficients, complex constant for defining transcendental equation

$c_{0}, T_{0} \quad$ ambient sound speed, mean static temperature

$D / D t, \vec{\nabla}, \nabla^{2}$ material derivative, 2D gradient operator, 2D Laplace operator

$d, L, H \quad$ depth of test liner, axial location of duct termination, height of duct ( 0.051 meters)

$E, M_{\text {ave }} \quad$ transcendental equation, average (uniform) flow Mach number

$f, \omega \quad$ excitation frequency, angular frequency

$I, k \quad$ index of summation, free space wavenumber

$i, n w a l l \quad$ unit imaginary number, number of wall measurement points

$L_{1}, L_{2} \quad$ leading edge of test liner, trailing edge of test liner

$K_{n}, \lambda_{n} \quad$ axial propagation constant, mode eigenvalue

$p_{0}, \rho_{0} \quad$ mean static pressure in duct, mean static density in duct

$N, M \quad$ number of points in axial direction, number of points in transverse direction

$p, u, v \quad$ acoustic pressure field, axial particle velocity, transverse particle velocity

$\vec{u}, \overrightarrow{u_{0}} \quad$ acoustic particle velocity vector, mean flow velocity vector

$x, y, z \quad$ transverse, spanwise, and axial coordinate

$t, z_{I} \quad$ time, location of a lower wall pressure measurement

$\gamma, \zeta \quad$ ratio of specific heats, normalized acoustic impedance

$\theta, \chi \quad$ normalized resistance, normalized reactance

$\{\Phi\}, \phi\left(\zeta_{\text {wall }}\right) \quad$ vector of unknown node coefficients, wall objective function

| |, $\quad$ absolute value of complex quantity, vector dot product

\footnotetext{
*Senior Research Scientist, Research and Technology Directorate, Computational AeroSciences Branch, Liner Physics Group, Mail Stop 128, Willie.R.Watson@NASA.Gov. AIAA Associate Fellow.

${ }^{\dagger}$ Senior Research Scientist, Research and Technology Directorate, Structural Acoustics Branch, Liner Physics Group, Mail Stop 463. Michael.G.Jones@NASA.Gov.AIAA Associate Fellow.

${ }^{\ddagger}$ Senior Research Scientist, Research and Technology Directorate, Structural Acoustics Branch, Liner Physics Group, Mail Stop 463, Tony.L.Parrott@NASA.Gov.
} 

Subscripts:
0 , wall mean flow quantity, wall quantity
ave, initial spatially averaged quantity, initial assigned value
s, exit source plane quantity, exit plane quantity
FEM, M quantity computed from finite element analysis, quantity measured on lower wall

\section{Introduction}

R ECEnTly, the NASA Langley Liner Physics Group conducted a detailed assessment of a number of propagation codes, one of which is used as the basis for the LaRC impedance eduction technique. This detailed assessment shed valuable light on potential causes for previously documented anomalous behavior; i.e., educed impedances that did not match the expected trends. This anomalous behavior has been observed with the single mode model, ${ }^{1}$ the convected Helmholtz model, ${ }^{2}$ and the linearized Euler model, ${ }^{3}$ and therefore does not appear to be caused by the choice of model. These anomalies have been observed at frequencies near anti-resonance and/or cut-on of higherorder modes. Although previous studies ${ }^{3}$ have documented potential explanations for the anomalous behavior, the root causes have not been fully investigated. The purpose of this paper is to launch an intensive investigation into the cause of the anomalous behavior. To this end, data are evaluated across wide frequency and Mach number ranges, such that detailed trend studies can be observed at frequencies near anti-resonance and/or cut-on of higher-order modes.

\section{Statement of Problem}

$\mathrm{T}$ HIS analysis considers acoustic wave motion through a uniform flow, constant area, rectangular duct as shown in Fig. 1. All walls of the duct (with the exception of the portion containing the liner) are considered rigid and impervious to sound. The lining is locally reacting and the unknown normalized impedance of the portion of the top wall containing the lining is denoted by $\zeta_{\text {wall. }}$ Throughout this report, all impedances are normalized with the characteristic impedance, $\rho_{0} c_{0}$, of the air flowing in the duct. A right-handed Cartesian coordinate system $(x, y, z)$ is used, with the $z$-axis pointing along the axis of the duct, the $y$-axis perpendicular to the duct sidewalls, and the $x$-axis perpendicular to the top and bottom walls. Consistent with most experimental apparatuses and aircraft engine nacelles, the source and termination planes are located in rigid wall sections of duct. The excitation frequency is assumed below cut-on of higher-order modes, so that the spanwise direction (i.e., the $y$ direction) supports only plane waves. Additionally, it is assumed that the acoustic pressure on the wall opposite the test liner, $p_{M}(z)$, is measured at a number of axial stations, $z=z_{I}$, and that the duct termination is near anechoic.

The problem at hand is to determine the unknown impedance of the test liner given a measurement of the source plane conditions, the acoustic pressure on the bottom wall opposite the test liner, the termination plane impedance, and the mean flow field. More specifically, it is desirable to thoroughly evaluate LaRC's two impedance eduction methodologies [the convected Helmholtz model $(\mathrm{CHE})^{2}$ and the linearized Euler model $(\mathrm{LEE})^{4}$ ] on the same liner, and over a range of frequencies and flow Mach numbers. The goal of this evaluation is to determine the source of the impedance eduction anomalies discussed in the previous section. This evaluation is based on a conventional, perforateover-honeycomb liner that is typical of that currently employed in high-bypass aircraft engine nacelles. Data used to perform this investigation are either synthesized from mode theory, or measured in the NASA Langley Research Center Grazing Incidence Tube (GIT).

\section{The CHE Impedance Eduction Model}

$\mathrm{T}^{\mathrm{H}}$

HE 2D CHE model is based on the solution to the 2D wave equation in a uniform flow

$$
\frac{1}{c_{0}^{2}} \frac{D^{2} p}{D t^{2}}=\nabla^{2} p, \quad \frac{D}{D t}=i \omega+\vec{u}_{0} \bullet \vec{\nabla}
$$

where a time dependence, $e^{i \omega t}$, has been assumed. In the current subsonic flow problem, the convected wave equation that governs the acoustic pressure field, i.e., Eq. (1), requires one boundary condition at the source boundary $(z=0)$, another at the duct termination $(z=L)$, and another at a rigid or acoustically lined wall. The source plane acoustic 
pressure is used as the source plane boundary condition

$$
p=p_{s}
$$

whereas, at the near anechoic duct termination, $z=L$, the boundary condition is written in the form ${ }^{2}$

$$
\frac{\partial p}{\partial z}=\frac{-i k p}{\zeta_{\text {exit }}+M_{\text {ave }}}
$$

When the termination is anechoic and located in a hardwall duct where only planar waves are cut-on, the measured value of $\zeta_{\text {exit }}$ is unity (i.e., $\zeta_{\text {exit }}=1$ ). However, when the termination in near anechoic and located in a hardwall section (e.g., as considered here) the termination impedance will vary slightly from unity. The GIT was designed to be as close to anechoic as possible, so that Eq. (3) is expected to represent a good approximation. Measurements of the exit impedance in the GIT, confirm that it is near anechoic, especially at the lower values of Mach number. It should be noted that Eq. (3) is also valid when a nonreflecting termination is located in a softwall duct. However, when the termination is located in a softwall duct, the exit impedance (i.e., $\zeta_{\text {exit }}$ ) may vary significantly from a value of unity. Several examples will be presented in the result section for which the termination is located in a softwall section of duct. However in theses examples, $\zeta_{\text {exit }}$ is synthesized from a mode solution. Finally, when the termination is highly reflecting but located in a hardwall duct where only plane waves are cut-on, Eq. (3) will not be valid but can be replaced by the more general condition

$$
\frac{\partial p}{\partial z}=-\frac{i k}{\zeta_{\text {exit }}}\left[\frac{1-M_{\text {ave }} \zeta_{\text {exit }}}{1-M_{\text {ave }}^{2}}\right] p
$$

In addition to Eq. (3), the use of the termination pressure

$$
p=p_{\text {exit }}
$$

as a boundary condition at the duct termination is also investigated. The third and final boundary condition required for the CHE model is the wall impedance boundary condition. When written in terms of the acoustic pressure, the wall impedance boundary condition is ${ }^{5}$

$$
-\frac{\partial p}{\partial x}=i k\left(\frac{p}{\xi_{\text {wall }}}\right)+2 M_{\text {ave }} \frac{\partial}{\partial z}\left(\frac{p}{\xi_{\text {wall }}}\right)+\frac{M_{\text {ave }}^{2}}{i k} \frac{\partial^{2}}{\partial z^{2}}\left(\frac{p}{\xi_{\text {wall }}}\right)
$$

It is noted that Eq. (6) is also used along the rigid wall portion of the duct wall. However, along a rigid portion of the duct wall, $\zeta_{\text {wall }}$ is set to infinity $\left(\zeta_{\text {wall }}=\infty\right)$.

\section{The LEE Impedance Eduction Model}

$\mathbf{I}^{\mathrm{N}}$ the 2D LEE model, the governing differential equations are the linearized equations governing conservation of Imass

$$
c_{0}^{2} \frac{D}{D t}\left(\frac{p}{c_{0}^{2}}\right)+\gamma p_{0} \vec{\nabla} \bullet \overrightarrow{u_{0}}+p \vec{\nabla} \bullet \overrightarrow{u_{0}}+c_{0}^{2} \gamma(\vec{u} \bullet \vec{\nabla}) \frac{p_{0}}{c_{0}^{2}}=0
$$

and momentum

$$
\gamma p_{0} \frac{D \vec{u}}{D t}+\gamma p_{0}(\vec{u} \bullet \vec{\nabla}) \overrightarrow{u_{0}}+p \frac{D \overrightarrow{u_{0}}}{D t}+c_{0}^{2} \vec{\nabla} p=0
$$

Here, the assumption is made that the acoustic process takes place homentropically in an ideal gas and that the fluid is inviscid. Note that Eq. (7) is a scalar equation and Eq (8) is a vector equation with two components in $2 \mathrm{D}$. The above acoustic system requires two boundary conditions at the source plane, one boundary condition at the duct termination, and a third boundary condition at an acoustically treated or rigid duct wall. ${ }^{6}$ At the source plane, the acoustic pressure and the transverse particle velocity component, $v$, are specified

$$
\left\{\begin{array}{l}
p \\
v
\end{array}\right\}=\left\{\begin{array}{l}
p_{s} \\
v_{s}
\end{array}\right\}
$$

At the duct termination, the boundary condition is specified in the form

$$
u=\frac{p}{\rho_{0} c_{0} \zeta_{\text {exit }}}
$$


and the wall impedance boundary condition is of the form ${ }^{5}$

$$
\rho c v=\left(1+\frac{M_{\mathrm{ave}}}{i k} \frac{\partial}{\partial z}\right)\left[\frac{p}{\zeta_{\text {wall }}}\right]
$$

Equation (11) is used at both a rigid and acoustically treated wall. However, at a rigid wall, the wall impedance is set to infinity (i.e., $\zeta_{\text {wall }}=\infty$ ).

\section{Numerical Solution for the Acoustic Field}

$\mathrm{T}$ HE numerical method chosen to obtain the solution to the acoustic field in the CHE and LEE models is the finite element method. Details of the implementation of the methodology are described in several papers ${ }^{2,4,7}$ and are not repeated herein. It is noted only that there are $N$ and $M$ evenly spaced nodes in the axial and transverse directions of the duct, respectively. Cubic Hermite polynomial basis functions are used to approximate the solution to the acoustic pressure field in the CHE model and a weak formulation is employed to incorporate the impedance and near anechoic boundary conditions. On the other hand, the LEE model uses linear elements to represent the acoustic pressure and particle velocity fields within each element, and the wall impedance and exit impedance boundary conditions are satisfied by constraining the nodal degrees of freedom. For both the CHE and LEE models, the finite element method results in a discrete system of equations of the form

$$
[A]\{\Phi\}=\{F\}
$$

where $[A]$ is a sparse, complex, asymmetric matrix whose order is $4 M N$ and $3 M N$, respectively, for the CHE and LEE models. Further, the vector $\{\Phi\}$ contains the unknown degrees of freedom at the nodes of the elements. Equation (12) is solved using an asymmetrical, parallel, direct sparse solver to obtain the unknown nodal parameters, $\{\Phi\}$. The solver uses a compressed column storage scheme to reduce storage overhead. Only the nonzero coefficients within $[A]$ are stored, along with two pointer arrays that store the column numbers and starting indices of these nonzero coefficients. To achieve efficient solutions, the sparse solver uses two accelerators: equation reordering to reduce fill during the factorization of $[A]$ and parallelization (i.e., the equation solver runs on multiple processors simultaneously). The finite element solution vector gives an approximation for the acoustic wall pressure opposite the test liner, $p_{\mathrm{FEM}}\left(z_{I}\right)$, for a specified wall impedance function, $\zeta_{\text {wall }}$. To avoid approximating the measured lower wall acoustic pressure, $p_{\mathrm{M}}\left(z_{I}\right)$, on the finite element grid, the finite element grid is chosen so that each measurement point, $z=z_{I}$, corresponds to a grid line in the finite element analysis. The finite element approximation to the acoustic wall pressure opposite the test liner, $p_{\mathrm{FEM}}\left(z_{I}\right)$, along with the measured values, $p_{\mathrm{M}}\left(z_{I}\right)$, provides the necessary information for the impedance eduction technique discussed in the following section.

\section{Impedance Eduction Technique}

G IVEN an initial estimate, $\zeta_{\text {wall }}=\zeta_{\text {initial }}$, for the wall impedance function, the finite element methodology is used to provide the values for the acoustic pressure [i.e., $p_{\mathrm{FEM}}\left(z_{I}\right)$ ] at measurement points on the wall opposite the test liner. Values of $\zeta_{\text {initial }}$ are then iteratively updated until a wall impedance function, $\zeta_{\text {wall }}$, is found that reproduces the measured wall pressure to within a specified tolerance. It is necessary, however, to automate the iterative procedure so that each new estimate for $\xi_{\text {initial }}$ is an improvement over its predecessor. We therefore implement an automated search procedure using an optimization algorithm. The objective function, $\phi\left(\zeta_{\text {wall }}\right)$, for the optimization algorithm is defined as the differences between the measured acoustic pressure on the wall opposite the test liner and that computed by the finite element method: ${ }^{7}$

$$
\phi\left(\zeta_{\text {wall }}\right)=\sum_{i=1}^{\text {nwall }}\left|p_{\mathrm{M}}\left(z_{I}\right)-p_{\mathrm{FEM}}\left(z_{I}\right)\right|
$$

where $n$ wall is the number of measurement points on the wall opposite the test liner. The optimization algorithm returns the value of the impedance function that minimizes the above objective function. It is a simple matter to show that the global minimum of the wall objective function, $\Phi\left(\zeta_{\text {wall }}\right)$, is the unknown impedance function of the test liner.

One of the most important aspects of the impedance eduction technique is the optimization algorithm. The optimization algorithm chosen was Stewart's adaptation of the Davidon-Fletcher Powell (SDFP) optimization algorithm that uses a finite difference approximation to the gradient of the objective function. ${ }^{8}$ This gradient-based optimizer has the disadvantage that it may converge to local optima (if they exist) and may become stuck in that portion of the 
impedance space where the objective function is extremely flat. However, these shortcomings are more than mitigated by the fact that SDFP tends to converge faster and give more accurate results than many of its competitors. Further, the occurrence of multiple local optima has to date not been observed with the impedance eduction process. However, since we are not aware of any definitive proof that multiple local optima cannot exist for this process, we continue to use multiple initial estimates $\left(\zeta_{\text {initial }}\right)$ to strengthen our confidence that the global optimum is achieved.

Recently, we have added a global-based optimizer, the "Genetic Algorithm," to our suite of optimization tools. Although the Genetic algorithm ${ }^{9}$ locates a global optimum, it tends to be much more computationally expensive because it performs a global search in the impedance plane. It was therefore used only sparingly in this study, and then only to spot-check the integrity of impedances educed using SDFP in the vicinity of where the anomaly occurs. In all cases, no noticeable differences between the impedance educed using SDFP and the Genetic Algorithm were observed. Therefore, only the SDFP results are presented.

\section{Results and Discussion}

$\mathrm{I}$ $\mathrm{N}$ this section, the impedance eduction methodologies are tested first on data synthesized from an exact mode solution and afterwards on measured data. These two examples illustrate whether the anomalies occur both with synthesized and measured data. Impedances are educed at twenty-seven excitation frequencies ranging from 0.4 to $3.0 \mathrm{kHz}$ in $0.1 \mathrm{kHz}$ increments. The impedance eduction codes use F90 Fortran with double precision (i.e., 64 bit) arithmetic and were designed to run in a multiprocessor environment using a shared memory implementation. This approach has been chosen because the primary hardware to be utilized was a Silicon Graphics, Altix 3700 system. In the current implementation the resistance, $\theta$, and reactance, $\chi$, of the test liner are the design variables $\left[\zeta_{\text {wall }}=\theta_{\text {wall }}+i \chi_{\text {wall }}\right]$. The optimizer, SDFP, runs only in sequential mode, using central finite differences to compute the gradient of the objective function, $\phi\left(\zeta_{\text {wall }}\right)$. The finite difference step size is $1 \times 10^{-8}$ and a stopping criteria of $\phi\left(\zeta_{\text {wall }}\right) \leq 1 \times 10^{-8}$ was used to terminate the search. The optimizer was constrained to realistic resistance values, $\theta_{\text {wall }} \geq 0.0$ and the duct geometry is that of the NASA Langley GIT ${ }^{2}$ (i.e., Fig. 1) for which $L=0.813$ meters, $L_{1}=$ 0.203 meters , and $L_{2}=0.610$ meters. The GIT has a square cross-section, so that the width (i.e., the distance from sidewall to sidewall) equals the duct height (i.e., the distance from bottom to top wall). The height of the GIT is 0.051 meters

\section{A. Synthesized Data}

In the first example, we use a liner 0.407 meters long $\left(L_{2}-L_{1}=0.407\right.$ meters) without a facesheet mass reactance. The specified impedance spectrum is

$$
\zeta_{\text {wall }}=\theta_{\text {wall }}-i \cot (k d)
$$

where a low resistance of $0.15\left(\theta_{\text {wall }}=0.15\right)$ is used and the depth of the cavity, $d$, is chosen such that the resonance frequency of the liner is at $1.5 \mathrm{kHz}$. These liner parameters were chosen because they produce an impedance spectrum similar to that of liners for which anomalies have occurred based on data acquired in the GIT. The input data used for this first example is synthesized from the exact mode solution for outgoing waves in an infinite duct using the liner model of Eq. (14). To simulate an infinite duct without reflections and a single outgoing mode, we remove the hardwall duct sections upstream and downstream of the liner and place the source and exit planes at $z=L_{1}$ and $z=L_{2}$, respectively. In the presence of the hard and softwall sections upstream and downstream of the liner, higher-order modes and reflections are generally present in the vicinity of near fields generated by the leading $\left(z=L_{1}\right)$ and trailing edge $\left(z=L_{2}\right)$ of the test specimen. For this reason the hardwall sections have been removed. The three sets of data needed to educe the impedance spectrum given in Eq. (14) using the CHE model are the source pressure $\left(p_{s}\right)$, the lower wall acoustic pressure $\left[p_{M}\left(z_{I}\right)\right]$, and the exit impedance, $\zeta_{\text {exit. }}$. These three sets of data were obtained from the mode solution

$$
p(z, x)=\sum_{n=1}^{\infty} A_{n} \cos \left(\lambda_{n} x\right) e^{-i K_{n} z}
$$

where $\lambda_{n}$ are complex zeroes of the transcendental function

$$
\begin{gathered}
E=\frac{i k \alpha_{n}}{\zeta_{\text {wall }}}-\lambda_{n} \tan \left(\lambda_{n} H\right) \\
\alpha_{n}=1-2\left(\frac{M_{\mathrm{ave}} K_{n}}{k}\right)+\left(\frac{M_{\mathrm{ave}} K_{n}}{k}\right)^{2}
\end{gathered}
$$


and the eigenvalue, $\lambda_{n}$, and axial propagation constant, $K_{n}$, are related by the dispersion relation

$$
\frac{K_{n}}{k}=\frac{M_{\mathrm{ave}} \pm \sqrt{1-\left(1-M_{\mathrm{ave}}^{2}\right)\left[\left(\lambda_{n} / k\right)^{2}\right]}}{-\left(1-M_{\mathrm{ave}}^{2}\right)}
$$

Note that for a given value of $\lambda_{n}$, the axial propagation constant $K_{n}$ satisfying Eq. (18) has two roots. Here, we will choose the root that corresponds to right-moving waves in the duct. It is easily shown that right-moving waves are identified as those modes for which the axial propagation constant, $K_{n}$, has a zero or negative imaginary part and a positive real part, respectively. The method of analysis described above requires a calculation of the mode eigenvalues, $\lambda_{n}$, that are the complex zeroes of the transcendental function, $E$. The method used to obtain these roots was the Newton-Raphson iterative scheme. From the mode solution in Eq. (15), the data required to educe the impedance spectrum in Eq. (14) was obtained. Only the least attenuated mode was allowed to propagate through the duct and the mode coefficient, $A_{n}$, was set at a $120 \mathrm{~dB}$ level with zero phase. A uniform spatial grid with 129 points in the axial direction $(N=129)$ and 21 points in the transverse direction $(M=21)$ was used to educe the impedance from the synthesized data.

Table 1

Average Mach number and mean static conditions for synthesized data

\begin{tabular}{rrr}
\hline$M_{\text {ave }}$ & $p_{0}$ & $T_{0}$ \\
\hline 0.000 & 101425.45 & 297.48 \\
0.085 & 101473.94 & 296.48 \\
0.253 & 101453.30 & 295.15 \\
0.401 & 101211.70 & 293.37 \\
\hline
\end{tabular}

The impedance spectra was educed (using the CHE model) for the four uniform flow Mach numbers, mean static pressures (in Pascals), and mean static temperatures (in Kelvin) given in table 1. The resistance and reactance spectra educed using the CHE model are compared to the exact spectra [see Eq. (14)] in Figs. 2 and 3, respectively. To the level of accuracy presented in Figs. 2 and 3, the educed impedance reproduce the exact impedance in Eq. (14) without any anomalies. We have also noticed that the small values of the finite difference step sizes and stopping criterion used in SDFP were also important parameters contributing to such excellent agreement between the educed and exact spectrum.

A major drawback introduced by the local, gradient-based optimizer (i.e., SDFP) is that it may converge to a local minimum if local minima exist. In this situation, different design points (i.e., educed impedances) may be obtained if the optimizer is initialized from different points in the design space. Because of reduced turnaround time resulting from usage of the parallel, sparse solver, an exhaustive evaluation of the effects of "starting location" (i.e., initial impedance) could be conducted. Several distinct starting locations were used and no local minima were found.

\section{B. Measured Data}

In the second example, input data required for the impedance eductions is obtained from measurements taken in the NASA Langley GIT. ${ }^{2}$ As described earlier, the CHE and LEE are 2D impedance eduction models. Thus, only data that is acquired at excitation frequencies below the "cut-on" of higher-order modes in the hard-wall section of the GIT should be expected to be properly described by these models. Due to the sound absorbing properties of the test liner, the possibility of higher-order modes cannot be avoided in the lined region. In the presence of the liner, higherorder modes and reflections are generally present in the vicinity of near fields generated by the leading $\left(z=L_{1}\right)$ and trailing edge $\left(z=L_{2}\right)$ of the test specimen. The test liner is a conventional perforate type liner composed of a punched aluminum face sheet bonded to a honeycomb core that was in turn bonded to a rigid back plate. The face sheet is $0.635 \times 10^{-3}$ meter thick with holes that were $0.991 \times 10^{-3}$ meters in diameter. The open area ratio is 0.087 . The honeycomb core has a cell size of $9.525 \times 10^{-3}$ meters and a depth of $38.100 \times 10^{-3}$ meters. Because the test liner was designed from homogeneous material, the impedance was assumed to be invariant over the length of the liner. The incident sound pressure level (SPL) is kept at a low excitation level (approximately $120 \mathrm{~dB}$ ) to minimize liner nonlinearity effects. Finally, the decision was made to present only zero flow results using the measured data. This is done to avoid processing measured data (obtained in the presence of a boundary layer) through impedance eduction 
codes that neglected the effects of the boundary layer. Flow results in the presence of measured data are currently being analyzed with the LEE model and will be reported at a later date.

Figure 4 compares the educed normalized impedance obtained from the CHE model using the baseline grid (i.e., $N=129$, and $M=21$ ) with that measured in the Langley Research Center Normal Incidence Tube (NIT). The educed normalized resistance and reactance spectra are plotted using a dual axis system, with the normalized resistance and reactance referenced to the left and right axis, respectively. The NIT results are used as the baseline against which to measure the accuracy of the GIT results because they are normally considered more accurate in the absence of grazing flow. However, NIT measurements cannot be made in the presence of grazing flow so GIT results are often relied upon. As expected, both the NIT and GIT normalized reactance show a $\cot (k d)$ type of behavior and the normalized resistance has a low value of approximately 0.15 (see Fig. 4). The NIT and GIT spectra are in very good agreement except at $0.4,0.5,2.8$, and $3.0 \mathrm{kHz}$. For example, the educed normalized resistance at $0.4 \mathrm{kHz}$ is lower than that measured in the NIT and the value at $0.5 \mathrm{kHz}$ is high (i.e., when compared to NIT measurements). Subsequently, the slope of the educed resistance curve in the region 0.4 to $0.5 \mathrm{kHz}$ is opposite in sign to that measured in the NIT. Further, the change in slope of the educed normalized resistance spectrum near $2.8 \mathrm{kHz}$ and rapid rise in its slope near $3.0 \mathrm{kHz}$ is not only surprising, but is not reproduced by the NIT measurements. This strange behavior at low frequency $(0.4$ and $0.5 \mathrm{kHz})$ and high frequency $(2.8$ and $3.0 \mathrm{kHz})$ has been observed in numerous other tests with different test liners and is referred to here as impedance eduction anomalies. Note that the anomalous behavior at 0.4 and $0.5 \mathrm{kHz}$ (see Fig. 4) occurs in the vicinity of the anti-resonant frequency on the low frequency end of the spectra, and the anomalous behavior at 2.8 and $3.0 \mathrm{kHz}$ occur in the vicinity of the anti-resonant frequency on the high end of the spectra.

To determine if the anomalous behavior observed in Fig. 4 was due to lack of grid refinement or a breakdown of the CHE model, the impedance spectrum was educed using the LEE model and a grid refinement study was performed on both models. Figure 5a shows the results of three different grid resolutions using the CHE model. Again the results are shown on a dual axis system with the normalized resistance and reactance reference to the left and right axis, respectively. The baseline grid, G0, has 129 evenly spaced points $(N=129)$ along the axis of the duct and 21 $(M=21)$ evenly spaced points in the vertical direction (i.e., between the top and bottom wall). The density of the second grid, G2, is obtained by doubling the density of the baseline grid. Finally, the third grid, G4, is obtained by doubling the density of the G2 grid. Thus, the G4 grid is four times denser (in each direction) than the baseline grid. Figure $5 \mathrm{~b}$ shows results of the grid refinement study on the LEE model. Both models are observed to have converged on the baseline grid for this impedance spectrum.

Figure 6 compares (using the dual axis system) the educed normalized impedances on the baseline grid for the CHE (Fig. 5a) and the LEE (Fig. 5b) models. Both models give educed impedances that are nearly identical (at least on the scale for which the results are plotted) and both models produce the anomalies at $0.4,0.5,2.8$, and $3.0 \mathrm{kHz}$. When one considers that

1. each model supports a different set of equations,

2. each model uses a different approximation to the acoustic pressure, and

3. each set of results are obtained using the SDFP optimization algorithm,

then the excellent comparisons between the models in Fig. 6 are indeed gratifying. Whereas the comparison between CHE and LEE in Fig. 6 is not an infallible basis for judging the accuracy of these models, it does show that the impedance eduction theory (as applied here) is not model dependent. Such a result is always a necessary step in assessing the source of the impedance eduction anomalies.

One potential explanation of the enigmatic behavior at frequencies close to an anti-resonant frequency (see Fig. 4) is the failure of the SDFP optimization algorithms. Figure 7a shows a contour map of the wall objective function, $\phi\left(\zeta_{\text {wall }}\right)$, at the lowest frequency (i.e., $0.4 \mathrm{kHz}$ ) where the anomaly occurs. The contour map was constructed using the CHE model and on the baseline spatial grid, G0. Here, 81 evenly spaced points along the normalized resistance axis and 81 evenly space points along the normalized reactance axis were used to construct the contour. The center of the eye of the contour (i.e., the optimum point) is located at $\xi_{\text {wall }}=0.07-3.72 i$. This corresponds to the value of the optimum impedance obtained using SDFP (Fig. 4). The diameter of the eye is approximately 0.1 dimensionless units and is larger than the educed normalized resistance of the test liner. Because the educed normalized impedance is the location of the center of the eye, then for the current low resistance liner, the unknown normalized impedance cannot be determined with better accuracy using the measured data. This conclusion is also supported by Fig. $7 \mathrm{~b}$ that compares the measured lower wall SPL (in decibels) and phase (in degrees) with that predicted by the CHE model. The results are plotted on a dual axis system with the lower wall SPL and phase reference to the left and right axis, 
respectively. The symbols in the figure are located at the 31 lower wall measurement points (i.e., $z=z_{I}$ ). Recall that at the optimum point, the lower wall SPL and phase returned by the CHE model should match those measured in the GIT. As shown in Fig. 7b, the lower wall SPL and phase predicted from the CHE model are well matched to the measured values. Although, the results are not shown for the sake of brevity, similar trends to that in Fig. 7a and Fig. $7 \mathrm{~b}$ were observed at $0.5 \mathrm{kHz}$.

As illustrated in Fig. 4, the discrepancy between NIT and GIT impedances are more prominent at the two highest frequencies ( 2.8 and $3.0 \mathrm{kHz}$ ). Figure 8a shows a contour map of the wall objective functions at $2.8 \mathrm{kHz}$. The eye of the contour is more diffused than at $0.4 \mathrm{kHz}$ and the diameter of the eye has doubled from that observed at $0.4 \mathrm{kHz}$ (see Fig. 7a). The optimum impedance from the contour map matches that from SDFP (see Fig. 4) quite well. Figure 8b shows a comparison of the measured lower wall SPL and phase (using the dual axis system) to that predicted from the CHE model. Although, the measured phases are well matched, significant differences are observed between the measured SPL and those predicted by the CHE model. These SPL differences are more prominent upstream of the leading edge of the liner (i.e., $z \leq 0.203$ meters) and in the vicinity of the near field produced by the leading edge of the liner.

Figure 9a shows a contour map of the wall objective function at $3.0 \mathrm{kHz}$. Differences between the normalized impedance measured in the NIT and that educed using the CHE model are the most prominent at this frequency. The eye is strongly diffused with a diameter of nearly 0.6 dimensionless units. A comparison of the measured lower wall SPL and phase with that predicted by the CHE model is given in Fig. 9b. The differences between the measured and predicted SPLs and phases are the most noticeable upstream of the leading edge of the liner (i.e., $z \leq 0.203$ meters) and in the vicinity of the near field produced by the leading edge of the liner. Much smaller eyes were observed at frequencies away from an anti-resonant frequency (i.e., where the anomalies do not occur). For example, Fig. 10a shows a contour map of the wall objective function at $2.5 \mathrm{kHz}$. This contour map is representative of what was observed at frequencies where the anomalies did not occur. Note that, in this case, the eye of the contour appears tightly wound with a much smaller diameter (approximately 0.03 dimensionless units) than is observed at frequencies close to an anti-resonance (i.e., $0.4,0.5,2.8$, and $3.0 \mathrm{kHz}$ ). Figure $10 \mathrm{~b}$ compares the measured lower wall SPLs and phases with that produced by the CHE model. Note that the measured SPLs and phases are well matched to those produced by the CHE model.

A brief study was conducted to investigate the potential sensitivity of the educed impedance to errors in the exit impedance. Of the input data parameters, the exit impedance boundary condition is of concern, at least in part because it is determined from acoustic pressure measurements with microphones not included in the objective function [see Eq. (13)]. Figure 11 show the educed normalized resistance obtained from the CHE model by decreasing the normalized exit resistance by 0.1 over the measured value (the curve with triangle symbols) and by increasing the measured normalized resistance by 0.1 (the curve with circles). This perturbation value was chosen because resistance variability of this amount has been observed in practice at selected frequencies. Note that at and around the frequencies close to an anti-resonant frequency (i.e., $0.4,0.5,2.8$, and $3.0 \mathrm{kHz}$ ) measurable changes in educed resistance are observed.

Having identified errors in the exit impedance as one potential source of the anomalies, two remedies are suggested to minimize this error source:

1. Allow the optimization algorithm to educe the normalized exit impedance by including the normalized exit resistance and normalized exit reactance as design variables in the optimization algorithm.

2. Replace the exit impedance boundary condition with an acoustic pressure boundary condition [Eq. (5)], such that the data used in the boundary condition are not independent from the remainder of the data used in the impedance eduction process.

Figure 12a shows the educed normalized resistance when each of the above remedies is implemented in the CHE model. Also plotted in the figure are the normalized resistances measured in the NIT and those educed using the exit impedance measured in the GIT. Note that the anomalies at 0.4 and $2.8 \mathrm{kHz}$ are reduced substantially (i.e., the educed resistances are much closer to the NIT measurements) when either of the suggested remedies are implemented. At $3.0 \mathrm{kHz}$, the suggested remedy of educing the exit impedance gives results closer to the NIT measurements than implementing the exit pressure boundary condition. On the other hand, at $0.5 \mathrm{kHz}$, implementing the exit pressure condition brings the educed resistance closer to the NIT measurements than the educed exit impedance approach, although, the improvement is quite modest. Figure $12 \mathrm{~b}$ shows the results for the educed normalized reactance. There is very little effect of either remedy on the educed reactance except at the highest frequency $(3.0 \mathrm{kHz})$. At $3.0 \mathrm{kHz}$, implementing the exit pressure condition reduces the high value of the educed normalized reactance obtained by using the measured exit impedance by 0.35 dimensionless units, whereas the reduction is only about 0.15 units using the educed exit impedance. Generally speaking, implementing the exit pressure boundary condition is observed to reduce 
the normalized impedance anomalies more than educing the exit impedance (Fig. 12a and Fig.12b). Figure 13a shows the contour map when the exit pressure boundary condition was implemented. Note that the diameter of the eye is now only fifty percent of the value obtained when the measured exit impedance was used to construct the contour map (see Fig. 9a). Figure 13b shows a comparison of the measured lower wall SPLs and phases to that predicted by the CHE model when the measured exit impedance is used and when the exit pressure is used as the terminating boundary condition. Note that there is improvement in the SPL and phase comparisons downstream of the trailing edge (i.e., $z \geq 0.610$ meters) of the liner but little improvement upstream of the leading edge (i.e., $z \leq 0.203$ meters).

While the impedance educed at $3.0 \mathrm{kHz}$ is improved by the exit pressure boundary condition, the educed impedance at this frequency remains an outlier in the educed impedance spectrum (i.e., when compared to the NIT measurements). Observe that $3.0 \mathrm{kHz}$ is very near anti-resonance, and is also approaching cut-on of the next higher-order mode. Since the liner attenuation is quite minimal near anti-resonance, differences in impedance such as that observed between the NIT and GIT results in Fig. 4 has traditionally not been of great concern. Further, it should also be noted that a near-evanescent mode is generated near the leading edge of the liner at a frequency approaching cut-on (e.g., $3.0 \mathrm{kHz}$ ), and this mode may survive to the source plane of the computational domain, because of a slow axial decay and the absence of acoustic treatment upstream of the leading edge of the test liner. This would result in a source plane that is not planar in the transverse direction (i.e., the $x$ direction) as assumed in the CHE and LEE models. The propagation of a high-order, near-evanescent mode, upstream to the source plane is one plausible explanation for the discrepancy between measurement and theory at the frequency closest to cut-on. One possible remedy for this would be to use the acoustic pressure profile across the source plane as the source boundary condition in the impedance eduction models. The GIT test window is designed to acquire these data, and this option may be pursued in future endeavors.

\section{Conclusions}

B ASED upon the results of this study, the following preliminary conclusions can be drawn:

1. In the current study, anomalous impedances were educed only at frequencies near anti-resonance or cut-on of higher-order modes.

2. Sensitivity of the educed impedance to small variations in the measured exit resistance is increased for these frequencies where anomalous impedances are educed.

3. This anomalous behavior occurs at identical frequencies for each of the impedance eduction models, whether based on the convected Helmholtz equation or the Linearized Euler equations.

(a) Grid refinement studies indicate the choice of grids is not the cause of these anomalies.

(b) Two optimization algorithms - one local and one global - provide similar anomalous results.

4. These anomalies are reduced by replacing the exit impedance boundary condition with an acoustic pressure boundary condition at the exit plane.

The results of this study offer evidence that, at least for the no-flow condition, it is important to properly model the termination boundary condition for the computation domain. It appears particularly important to note that the common usage of an anechoic, plane wave termination boundary condition in ducts where the "true" termination is reflective, may act as a trigger for anomalous results. The effects of mean flow on this "conclusion" need to be evaluated and this effort is currently underway.

\section{References}

\footnotetext{
${ }^{1}$ Jones, M. G., Watson, W. R., Tracy, M. B., and Parrott, T. L., "Comparison of Two Acoustic Waveguide Methods for Determining Liner Impedance," Proceeding of the 7th AIAA/CEAS Aeroacoustics Conference and Exhibit, AIAA 2001-2266, Maastricht, The Netherlands, May 28-30, 2001.

${ }^{2}$ Watson, W. R., Jones, M. G., and Parrott, T. L., "Validation of an Impedance Eduction Method in Flow," AIAA Journal Vol. 37, No. 7, July 1999, pp. 818-824.

${ }^{3}$ Watson, W. R. and Jones, M. G., "Comparison of Convected Helmholtz and Euler Model for Impedance Eduction in Flow," AIAA Paper 2006-2643, Proceedings of the 12th AIAA/CEAS Aeroacoustics Conference \& Exhibit, Cambridge, MA, May, 2006.

${ }^{4}$ Watson, W. R., Tracy, M. B., Jones, M. G., and Parrott, T. L., "Impedance Eduction in Shear Flow," AIAA Paper 2001-2263, Proceedings of the 7th AIAA/CEAS Aeroacoustics Conference \& Exhibit, Maastricht, The Netherlands, May 28-30, 2001.

${ }^{5}$ Myers, M. K., "On the Acoustic Boundary Condition in the Presence Of Flow," Journal Of Sound And Vibration, Vol. 71, No. 3, 1980, pp. $429-434$
} 

1987.

${ }^{6}$ Thompson, K.W., "Time Dependent Boundary Conditions for Hyperbolic Systems," Journal of Computational Physics Vol. 68, pp. 1-28,

${ }^{7}$ Watson, W., Jones, M., and Parrott, T., " A Quasi-3-D Theory for Impedance Eduction in Uniform Grazing Flow," AIAA Paper 2005-2848, Proceedings of the 11th AIAA/CEAS Aeroacoustics Conference \& Exhibit, May 23-25, 2005, Monterey, CA.

${ }^{8}$ Stewart, G. W. III "A Modification of Davidon's Minimization Method to Accept Difference Approximations of Derivatives," Journal of ACM, Vol. 14, No. 1, 1967, pp 72-83.

${ }^{9}$ Goldberg, D., Genetic Algorithms in Search, Optimization and Machine Learning, Addison-Wesley, New York, NY, 1989 , pp. 75-147.

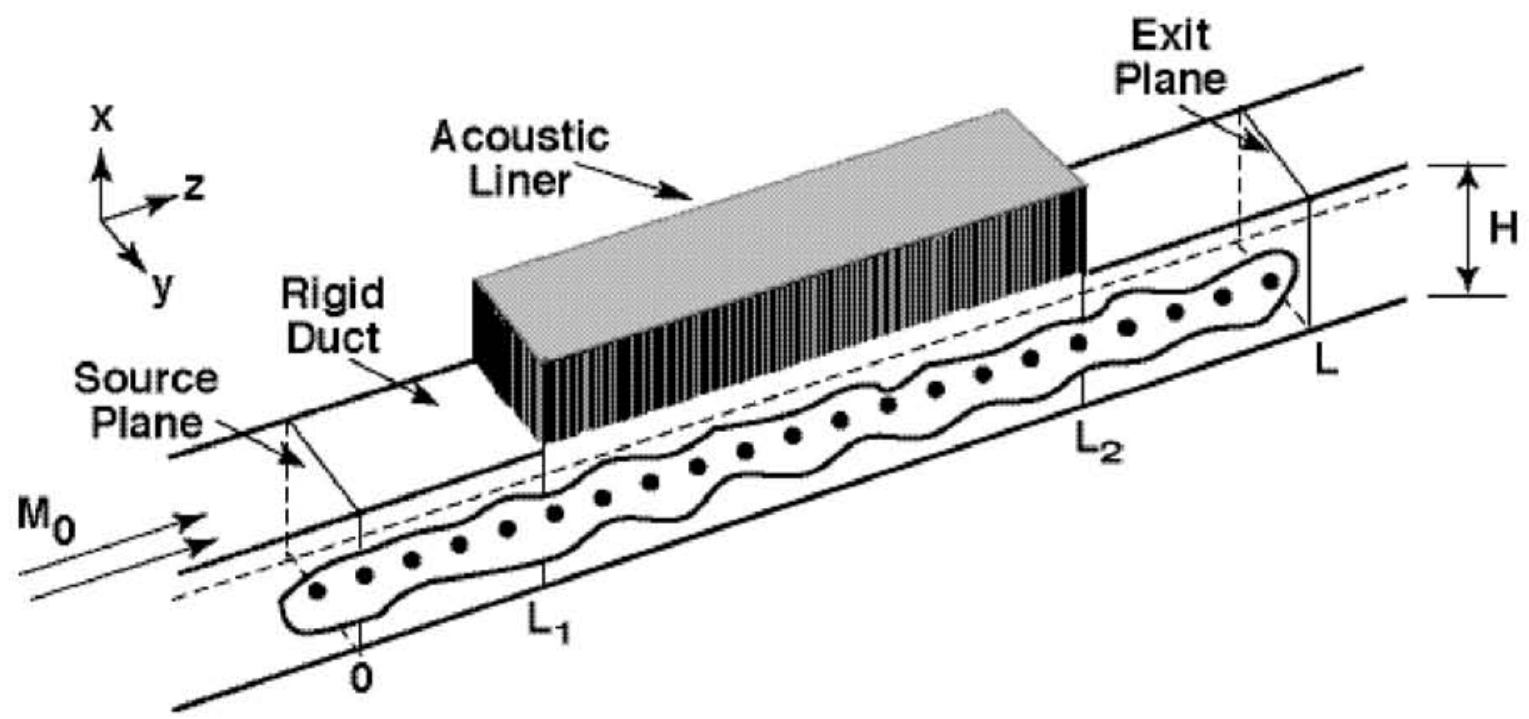

Fig 1. 3D Duct and Cartesian coordinate system. 


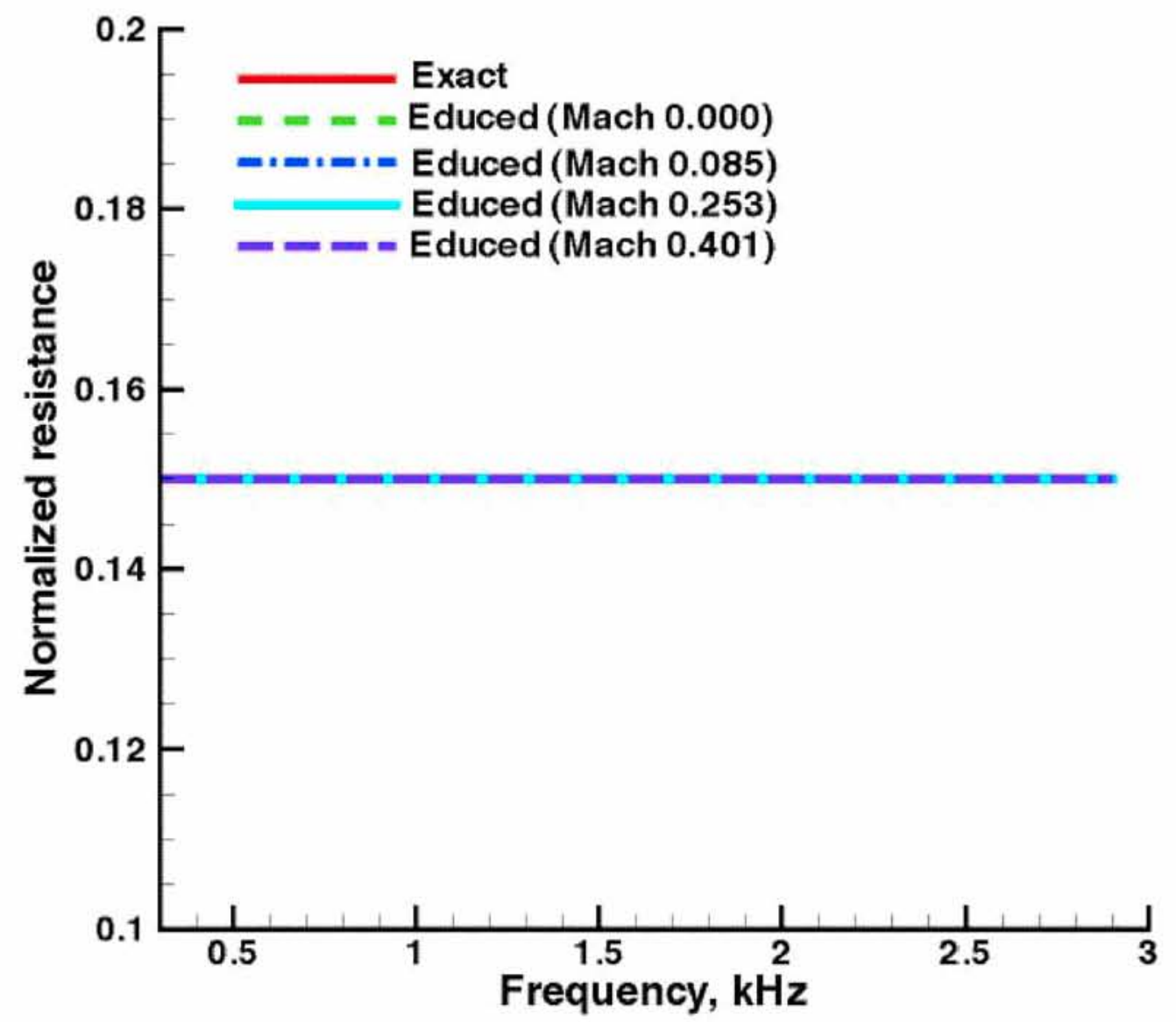

Fig 2. Exact and educed resistance from CHE model using synthesized data: shows no anomalies. 


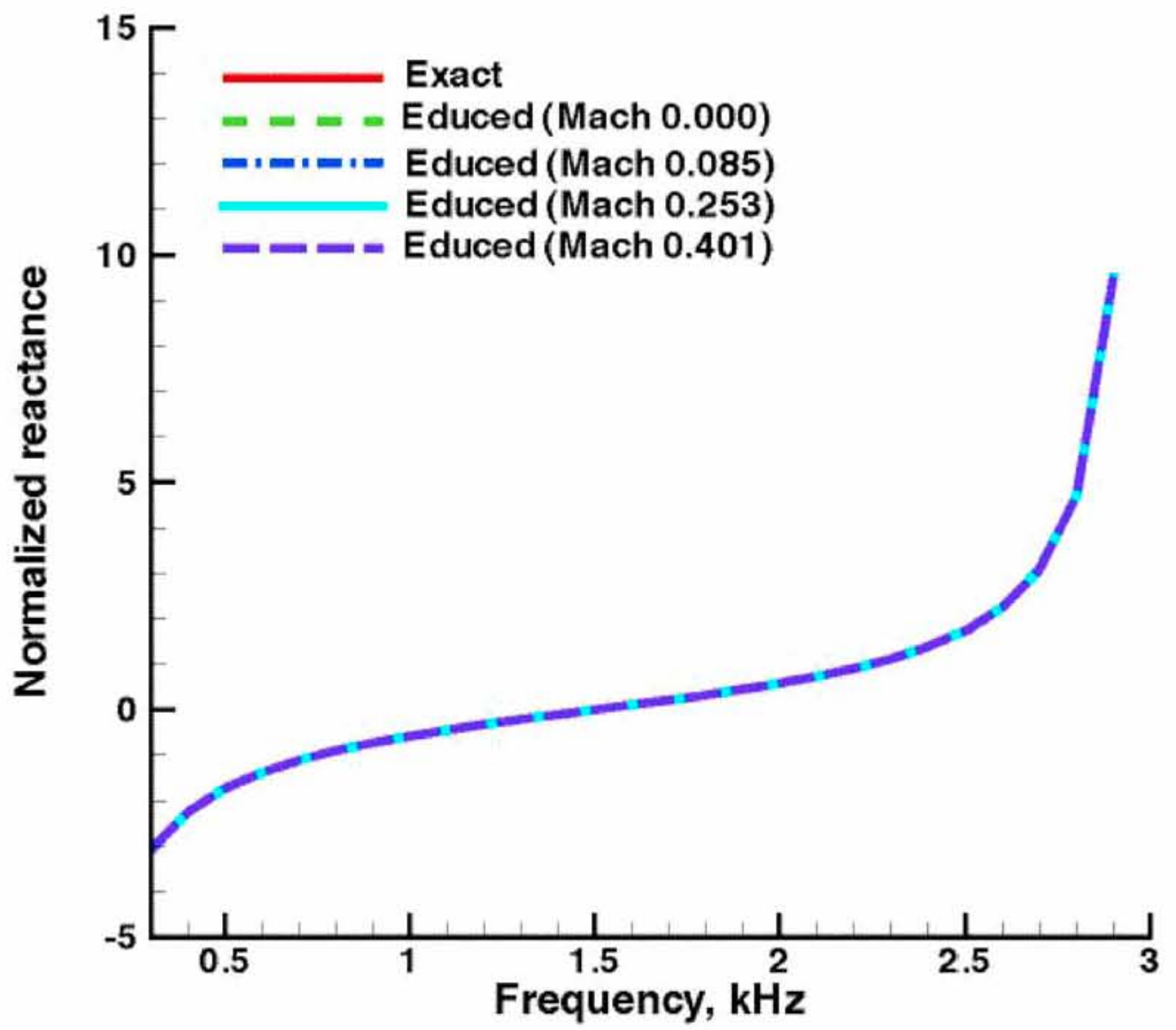

Fig 3. Exact and educed reactance from CHE model using synthesized data: shows no anomalies. 


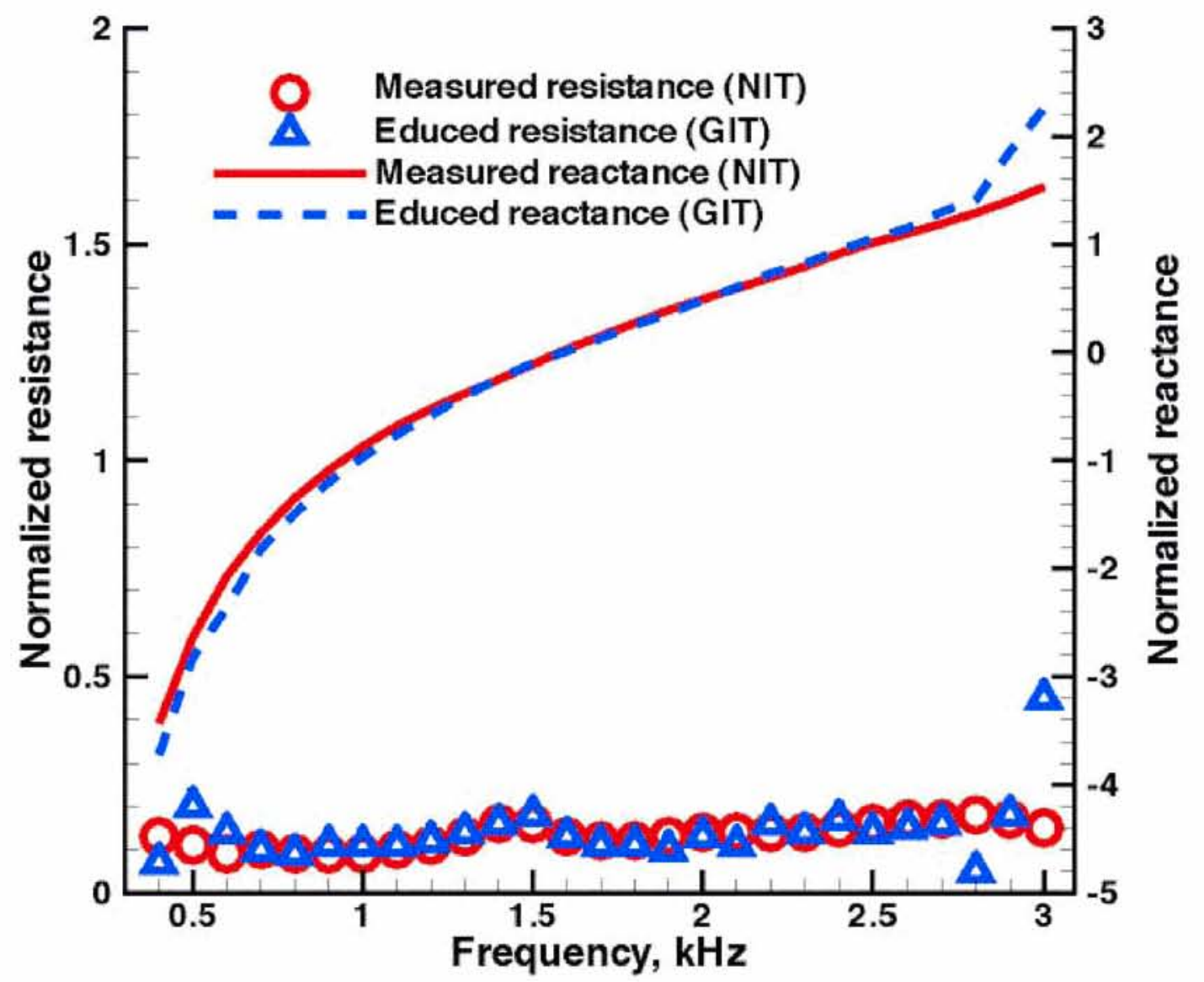

Fig 4. Comparison of the educed normalized impedance from the CHE model using measured data to normal incidence tube (NIT) measurements without flow: shows anomalies at frequencies close to anti-resonance $(0.4,0.5$, 2.8, and 3.0 $\mathrm{kHz}$ ). 


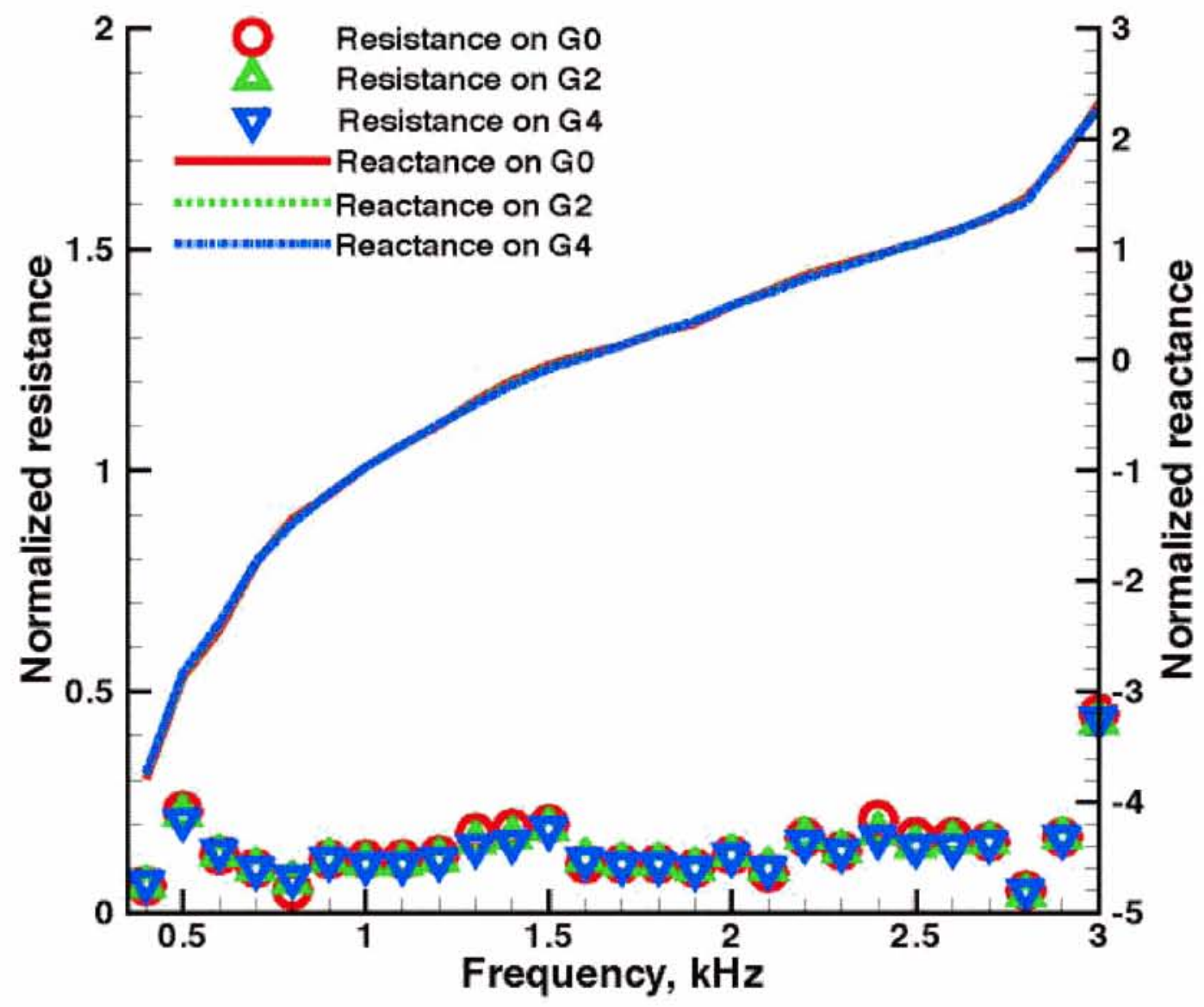

Fig 5a. Educed normalized impedance spectra produced by the CHE model on three grids (G0, G2, and G4) using measured data and no flow: shows the invariance of the educed normalized impedance spectra to the change in grid resolution. 


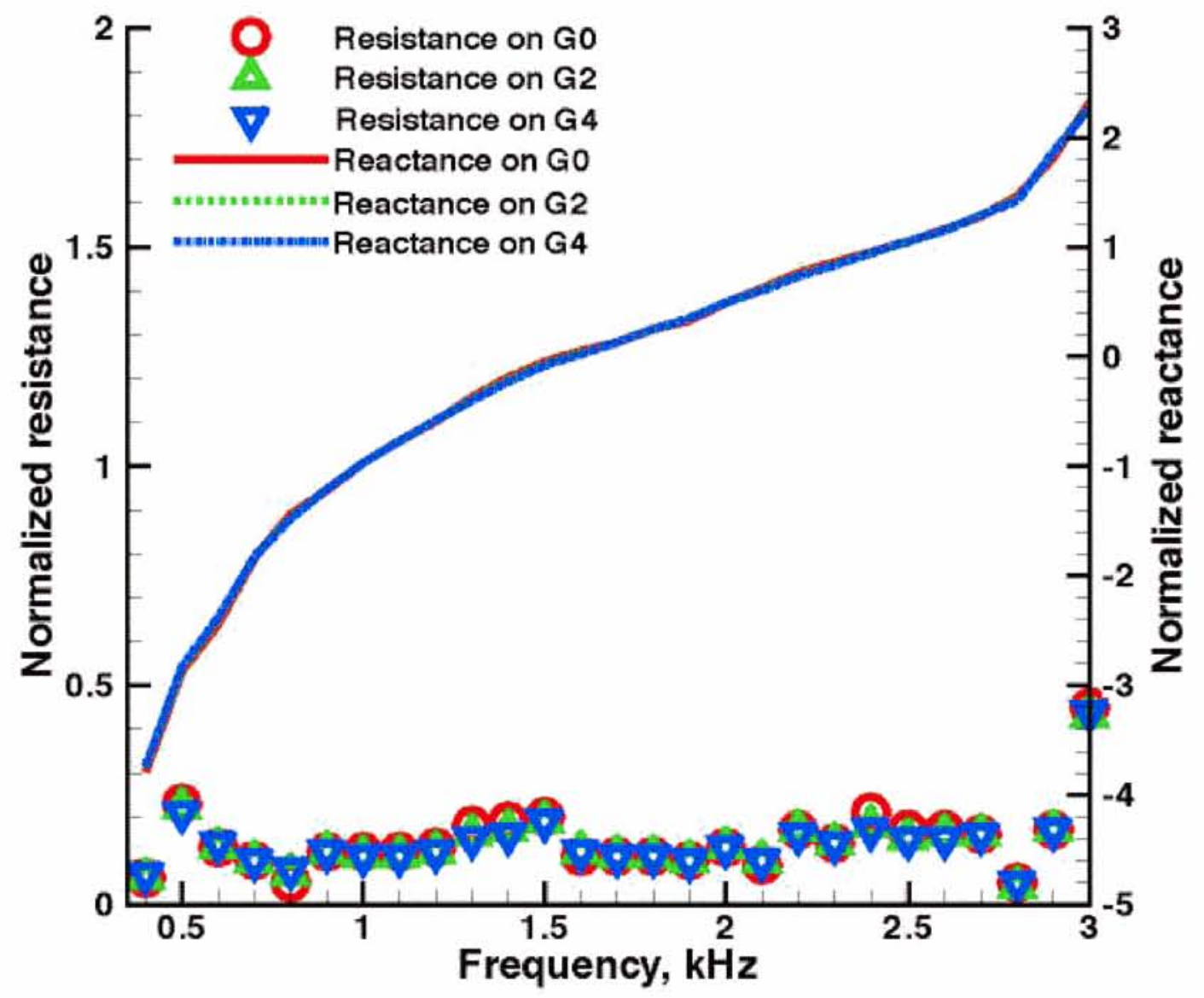

Fig 5b. Educed normalized impedance spectra produced by the LEE model on three grids (G0, G2, and G4) using measured data and no flow: shows the invariance of the educed normalized impedance spectra to change in grid resolution. 


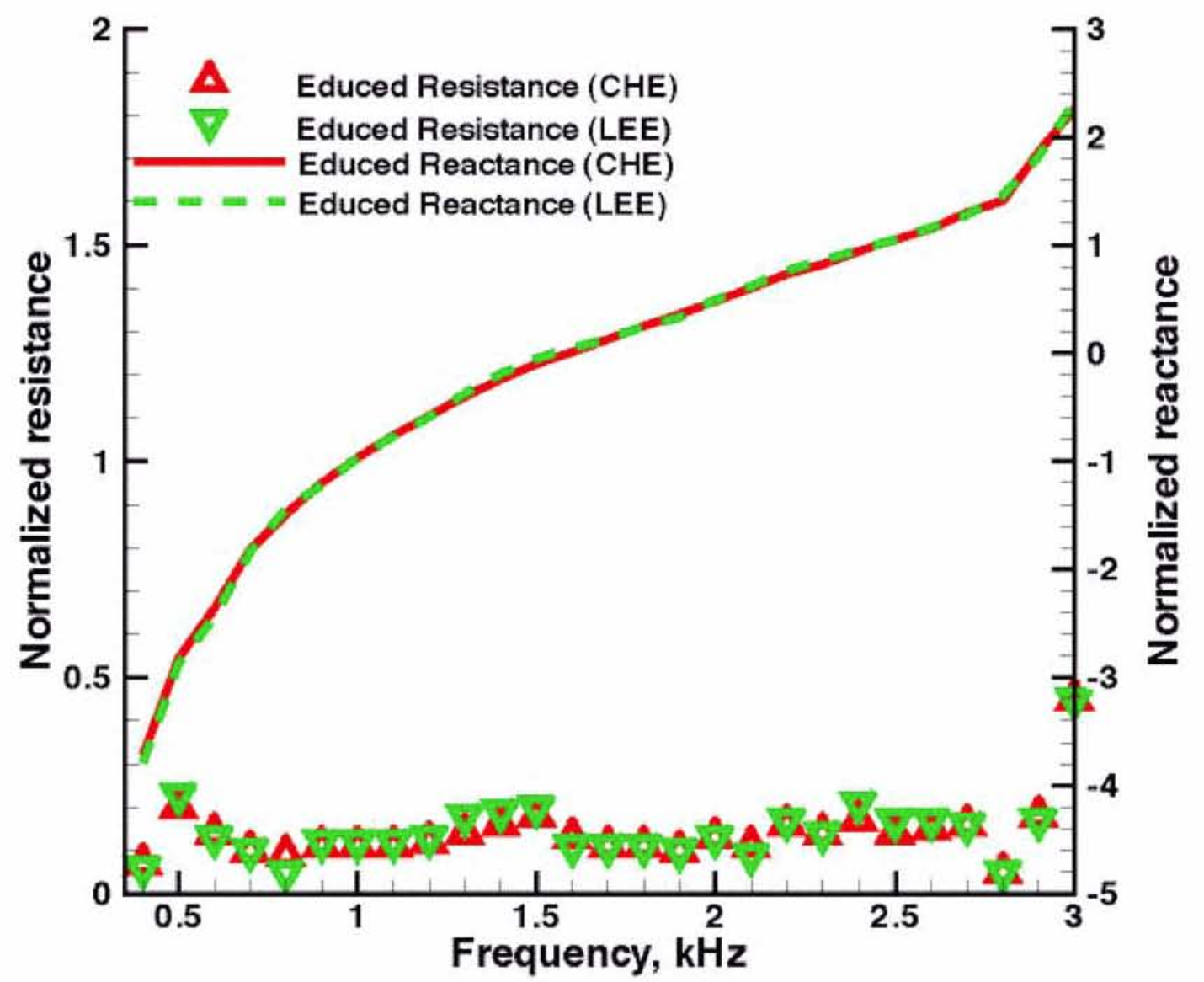

Fig 6. Comparison of educed normalized impedance spectra obtained from the CHE and LEE model using measured data on the baseline grid, G0, without flow: shows convergence of the CHE and LEE models. 


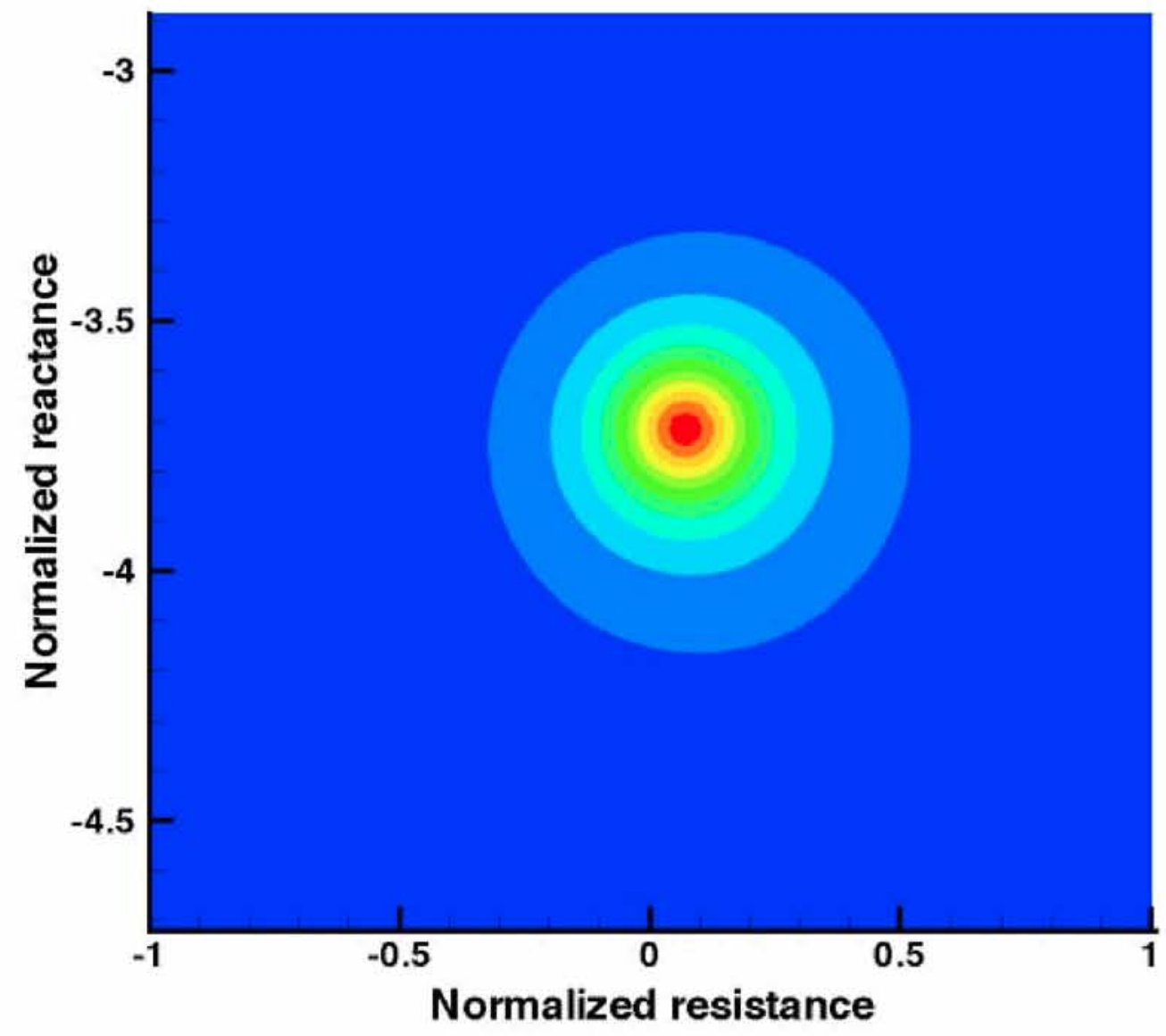

Fig 7a. Contour map of the wall objective function produced by the CHE model at $0.4 \mathrm{kHz}$ using measured data and no flow: shows that the optimum point of the contour map equals that produced by SDFP. 


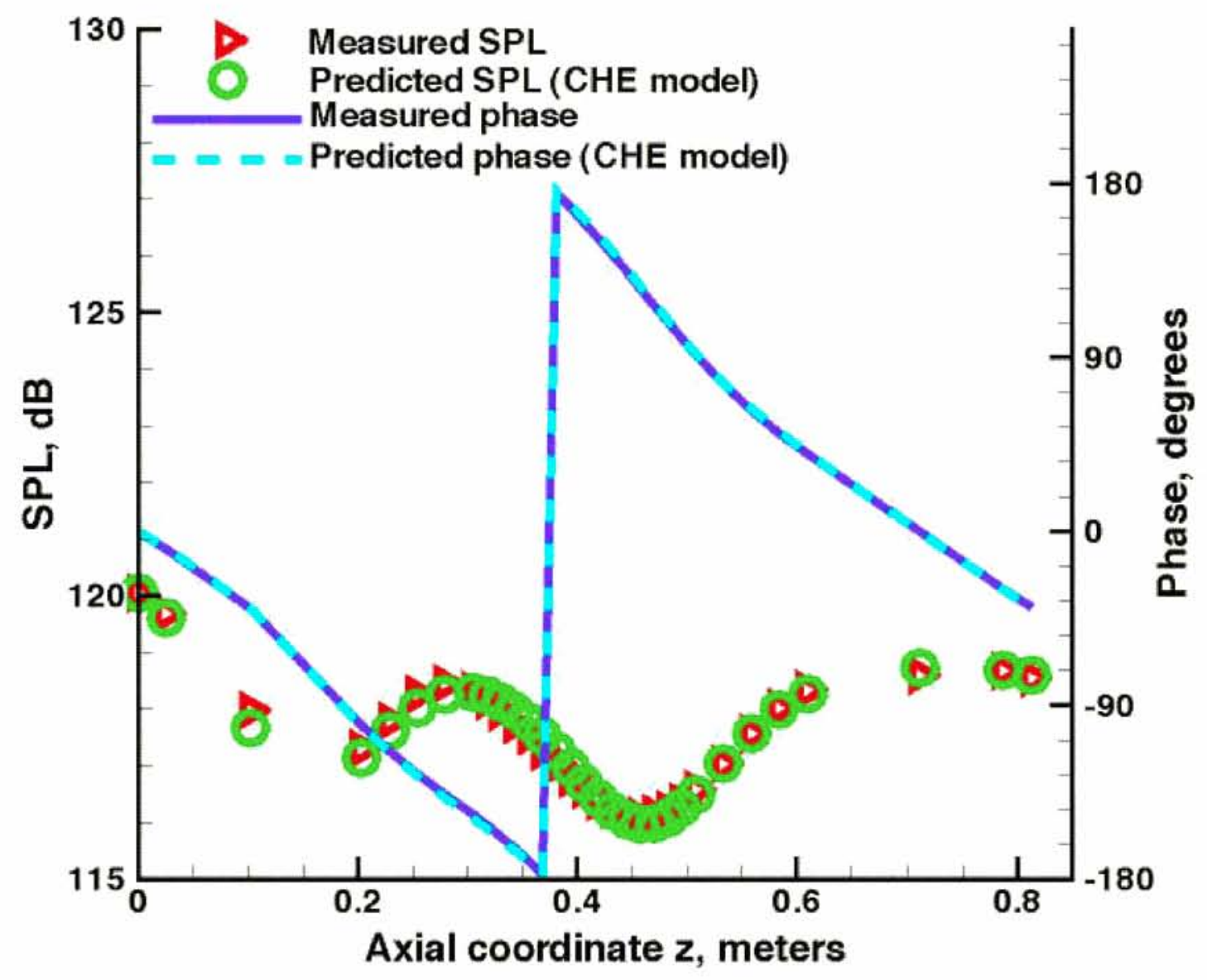

Fig $7 \mathrm{~b}$. Comparison of the measured lower wall sound pressure level (SPL) and phase at $0.4 \mathrm{kHz}$ to that predicted by the CHE model using measured data and no flow: shows a good match of measured and predicted SPL and phase. 


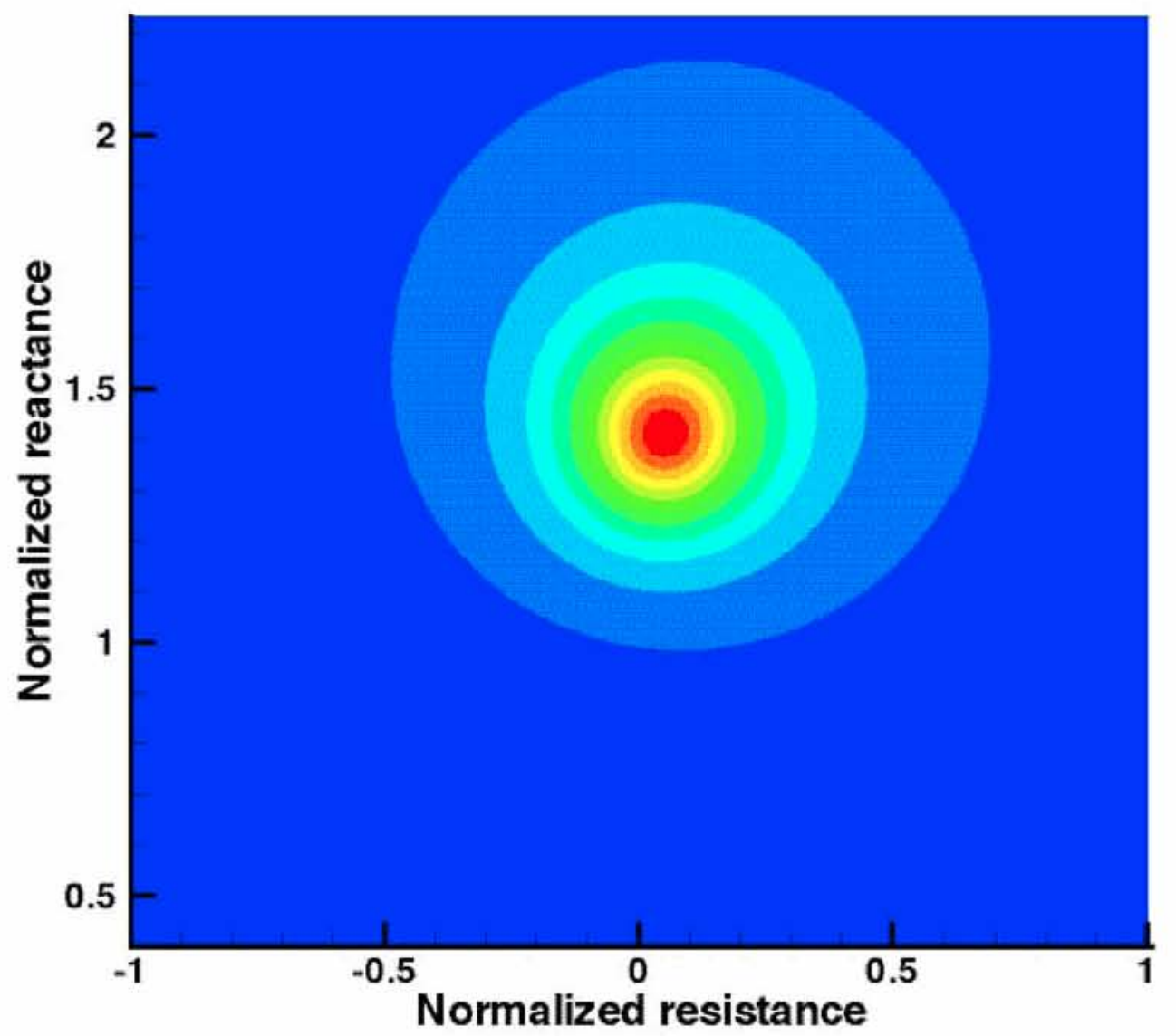

Fig 8a. Contour map of the wall objective function predicted by the CHE model at $2.8 \mathrm{kHz}$ using measured data and no flow: shows a moderate size eye. 


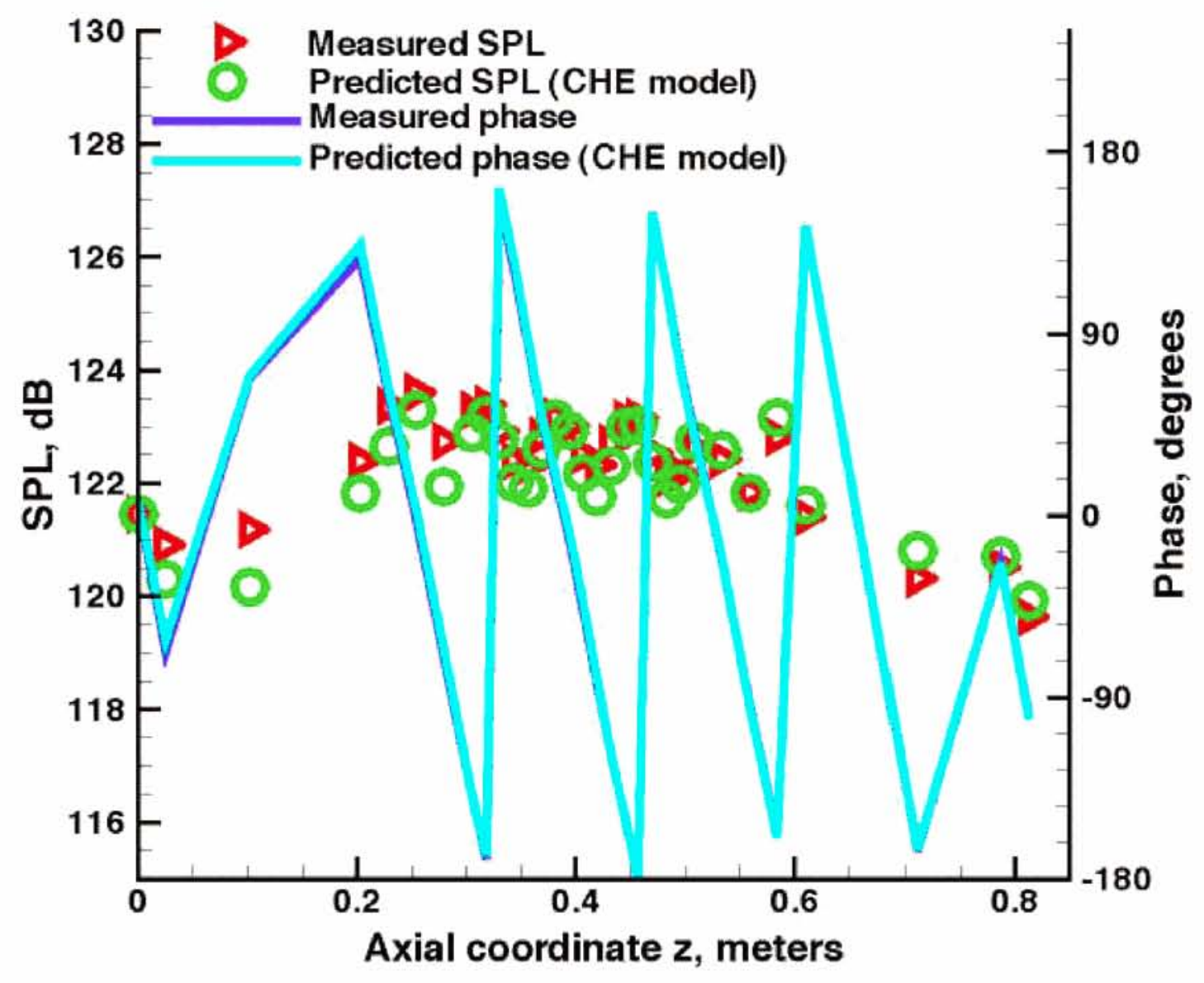

Fig 8 b. Comparison of the measured lower wall sound pressure level (SPL) and phase at $2.8 \mathrm{kHz}$ to that predicted by the CHE model using measured data and no flow: shows SPL errors upstream of the leading edge of the liner and in the vicinity of the near field produced by the leading edge of the liner. 


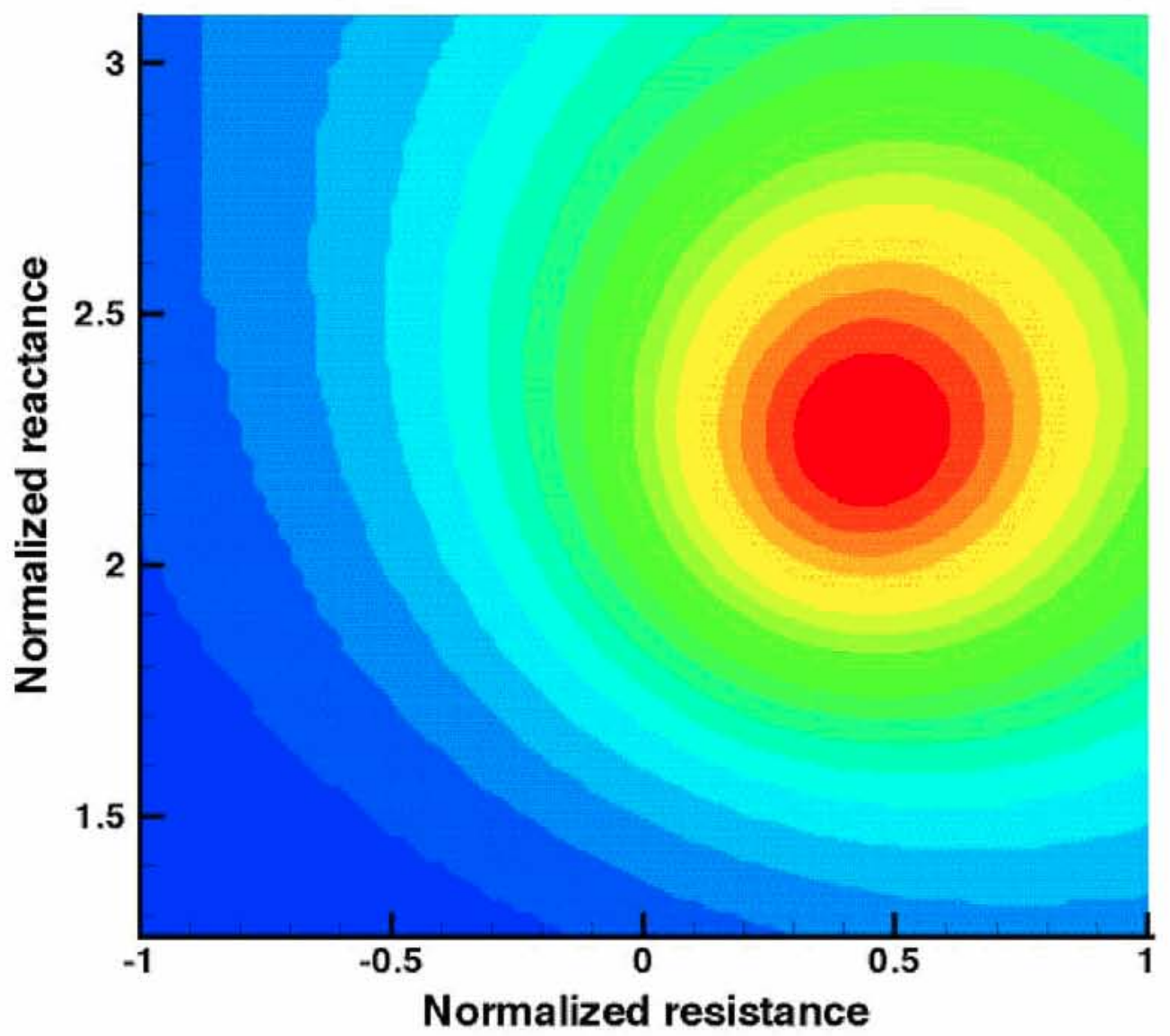

Fig 9a. Contour map of the wall objective function predicted by the CHE model at $3.0 \mathrm{kHz}$ using measured data and no flow: shows an extremely large eye. 


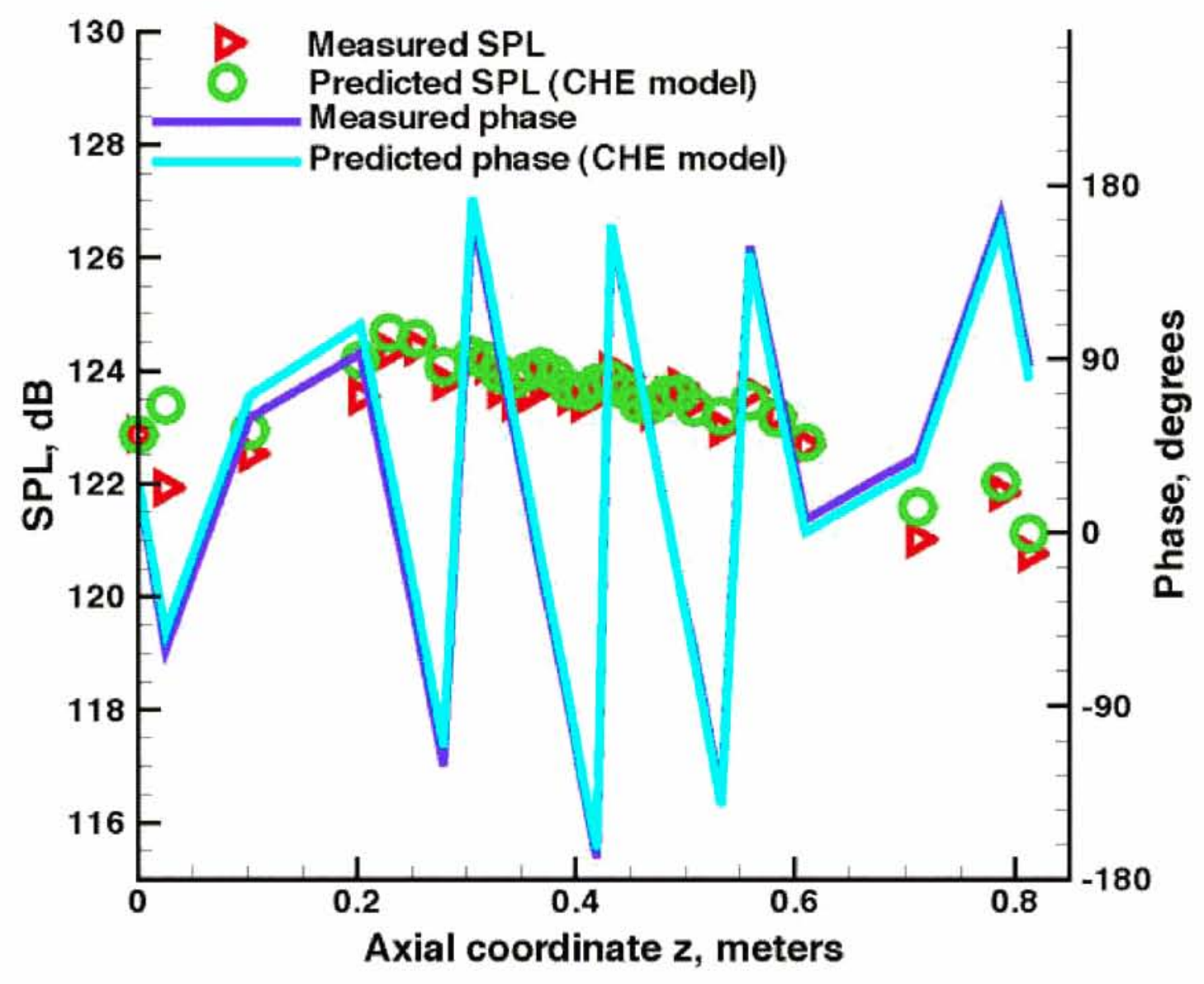

Fig 9b. Comparison of the measured lower wall sound pressure level (SPL) and phase at $3.0 \mathrm{kHz}$ to that predicted by the CHE model using measured data and no flow: shows SPL and phase errors upstream of the leading edge of the liner and in the vicinity of the near field produced by the leading edge of the liner. 


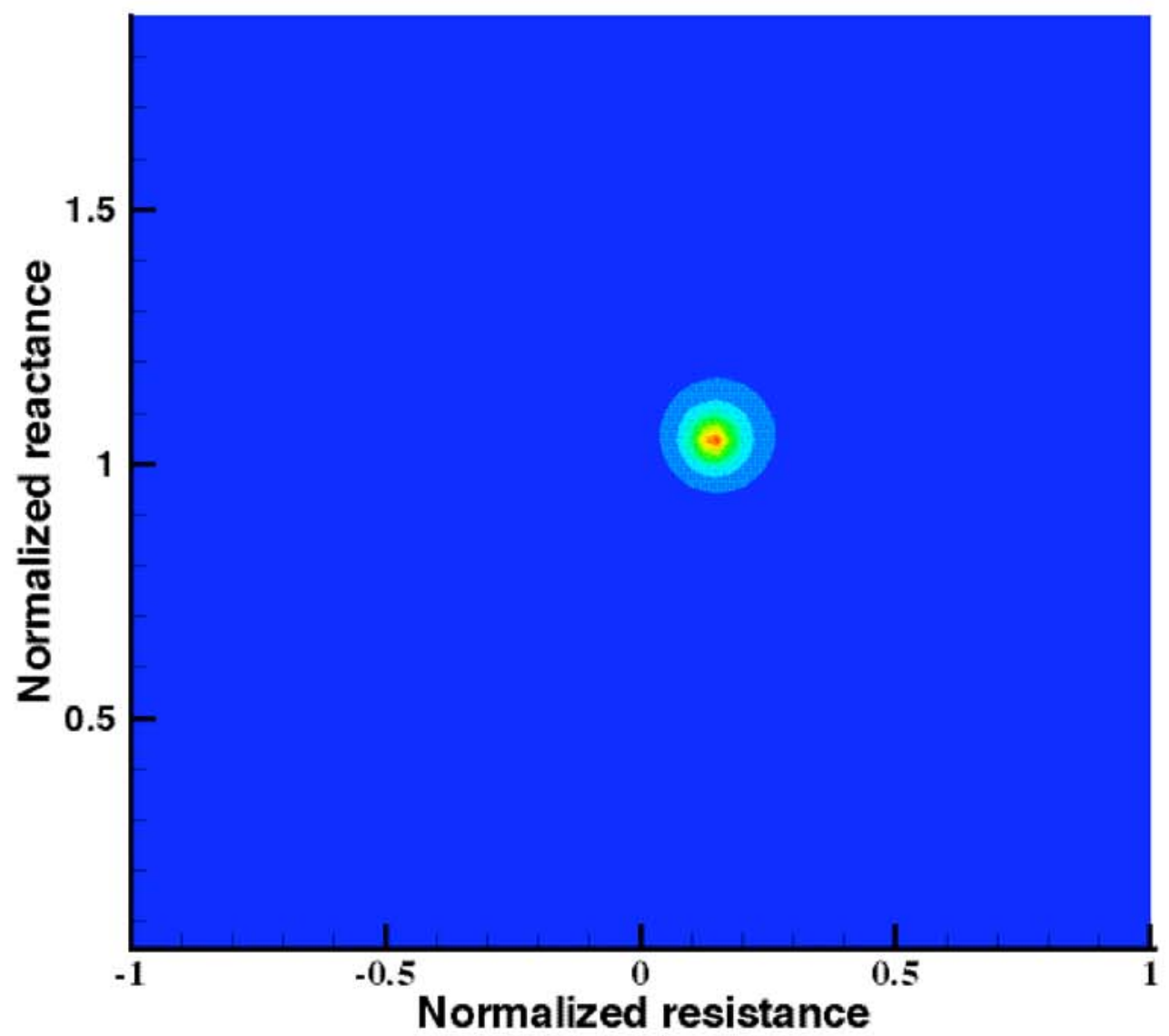

Fig 10a. Contour map of the wall objective function predicted by the CHE model at $2.5 \mathrm{kHz}$ using measured data and no flow: shows an extremely small eye. 


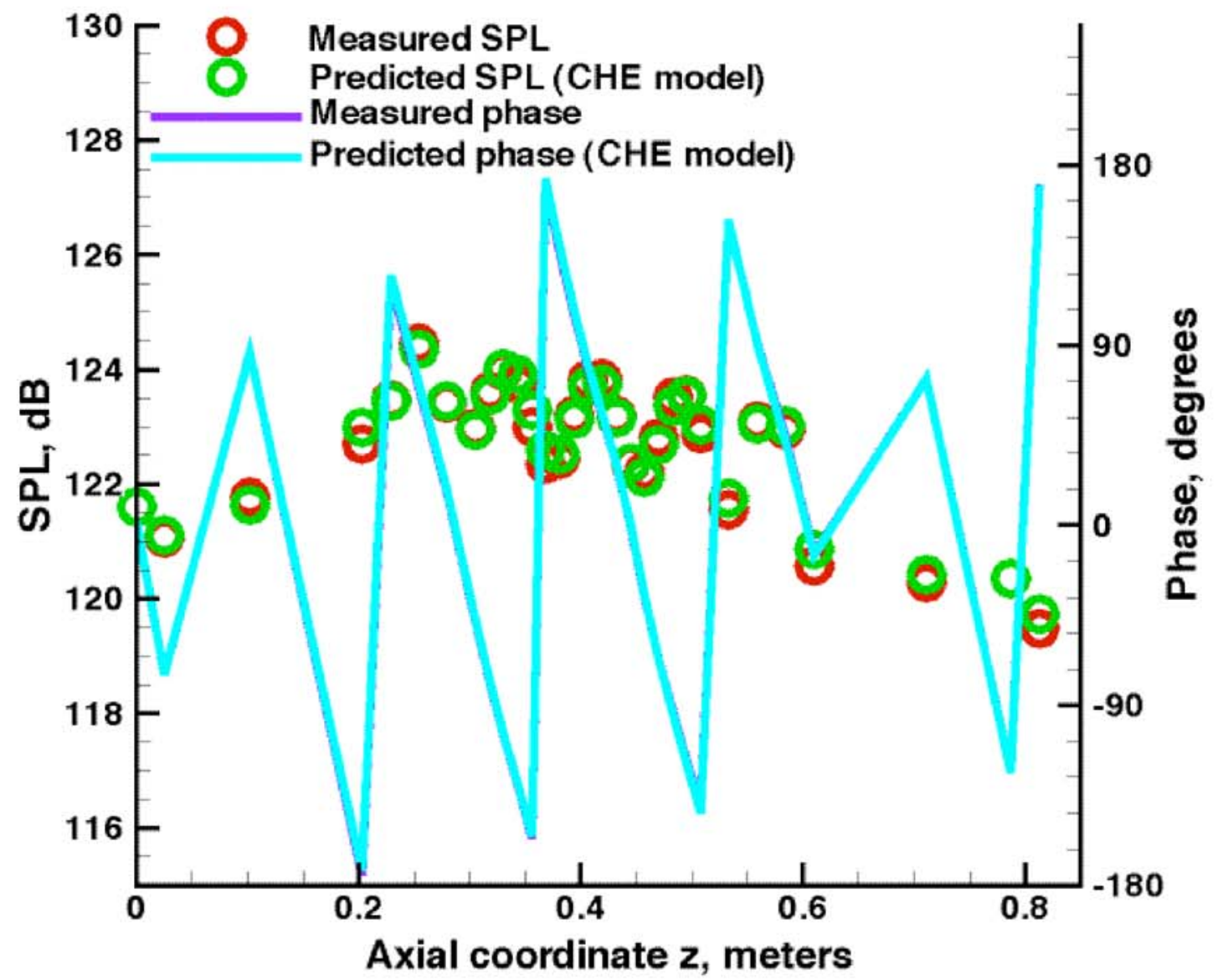

Fig 10b. Comparison of the measured lower wall sound pressure level (SPL) and phase at $3.0 \mathrm{kHz}$ to that predicted by the CHE model using measured data and no flow: shows a good comparison of SPL and phase. 


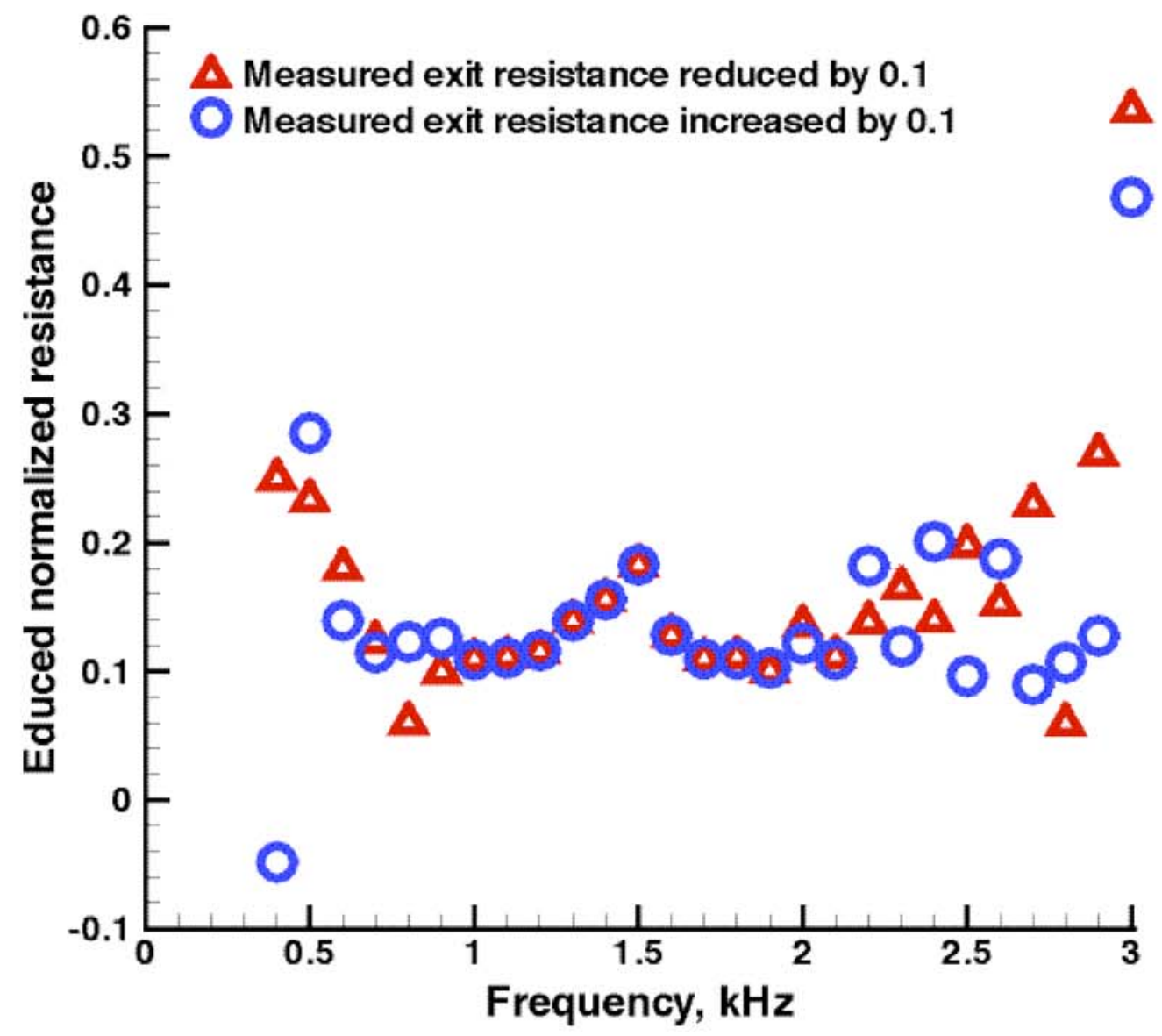

Fig 11. Effects of exit impedance resistance errors of \pm 0.1 on the educed resistance using the CHE model, measured data, and no flow: shows sensitivity of the educed normalized resistance to measured exit resistance errors at and near the anomalous frequencies $0.4,0.5,2.8$, and $3.0 \mathrm{kHz}$. 


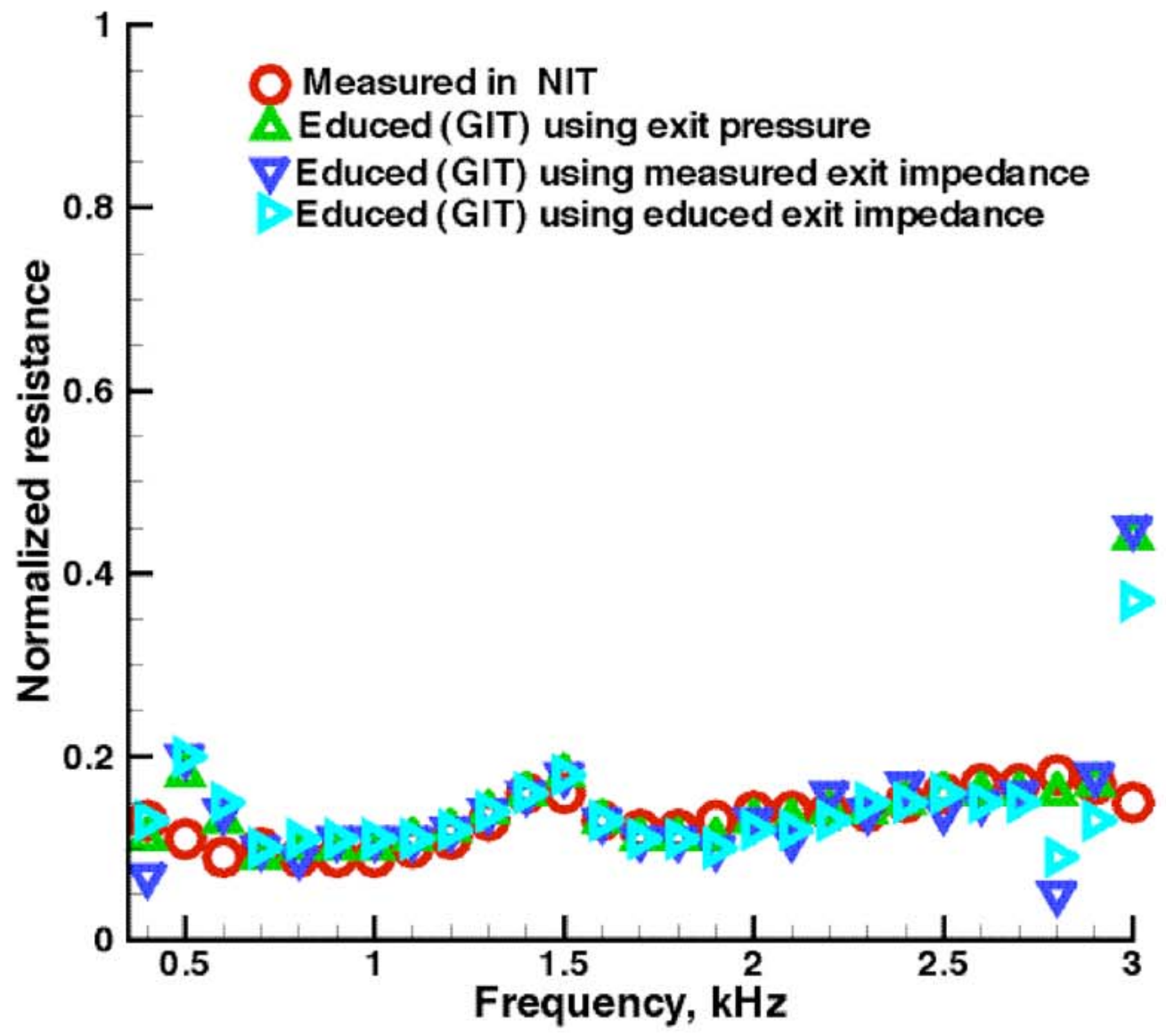

Fig 12a. Educed normalized resistance using the exit pressure, the exit impedance, and the educed exit impedance boundary condition using the $\mathrm{CHE}$ model, measured data, and no flow: shows a reduction in the anomalies at $0.4,2.8$, and $3.0 \mathrm{kHz}$ using the educed impedance and exit pressure boundary condition. 


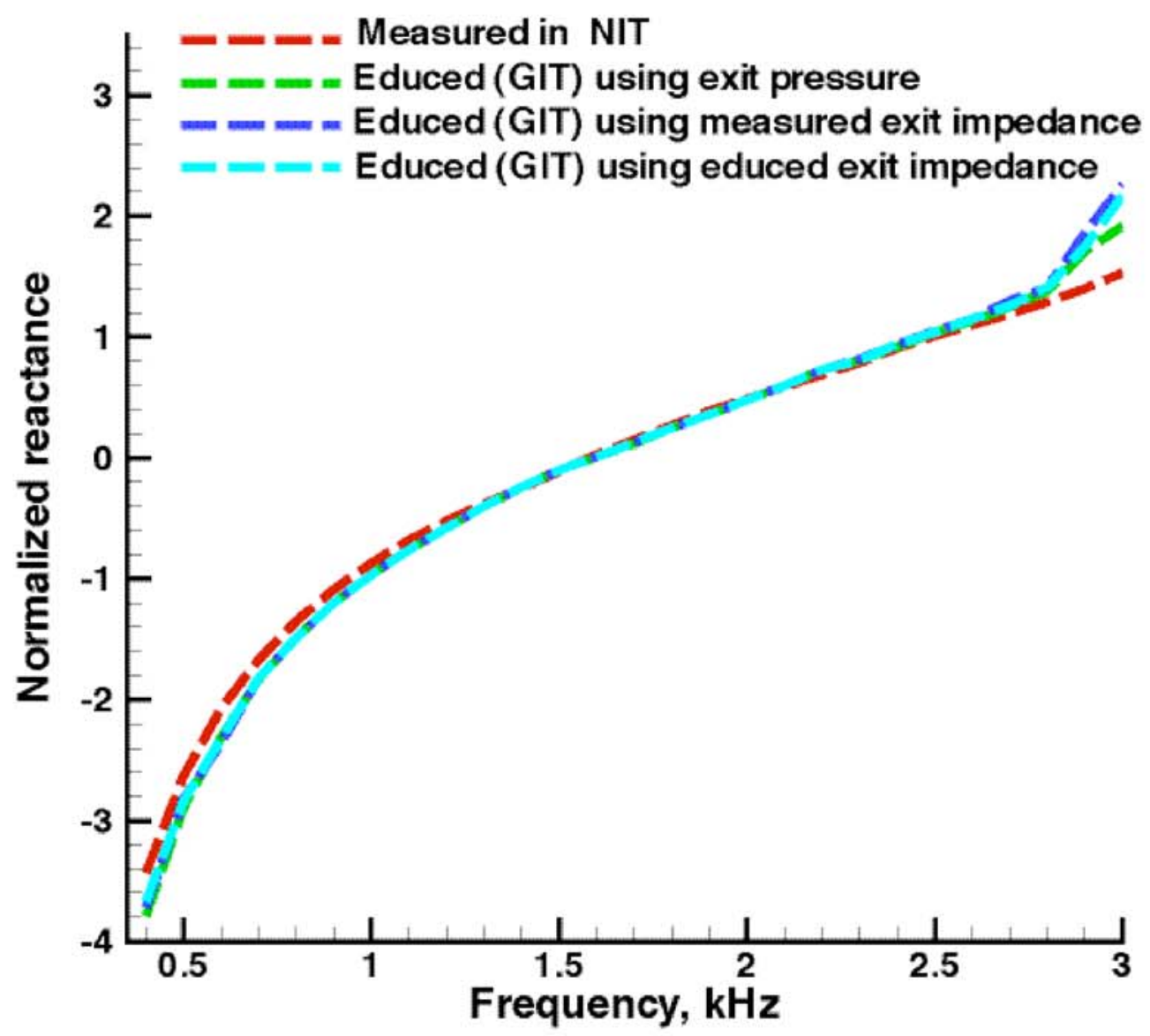

Fig 12b. Educed normalized reactance using the exit pressure, the exit impedance, and the educed exit impedance boundary condition using the CHE model, measured data, and no flow: shows a reduction in the anomaly at $3.0 \mathrm{kHz}$. 


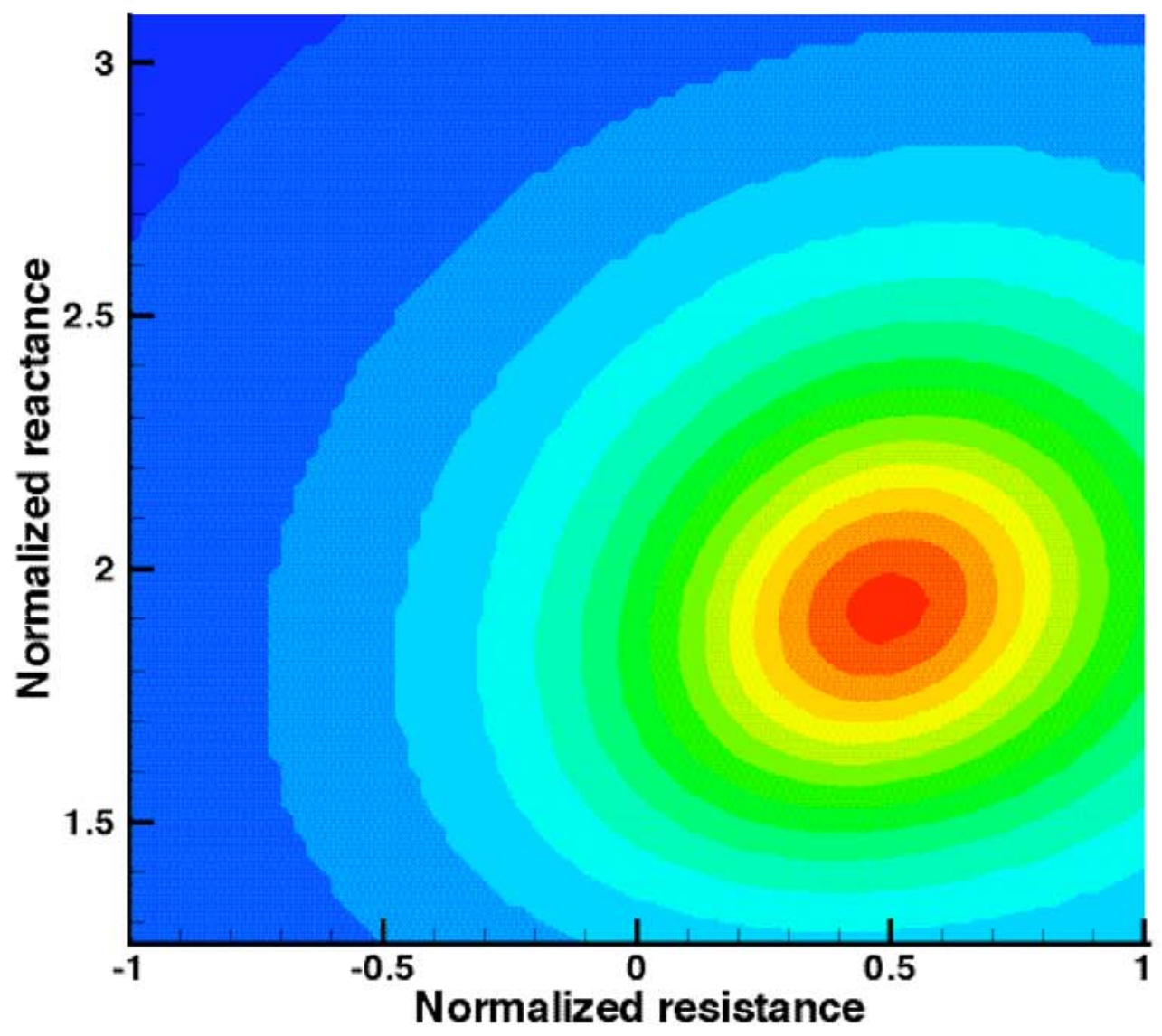

Fig 13a. Contour map of the wall objective function predicted by the CHE model at $3.0 \mathrm{kHz}$ using measured data, the exit pressure boundary condition, and no flow: shows a fifty percent reduction in the size of the eye compared to the exit impedance boundary condition (see Fig. 9a). 


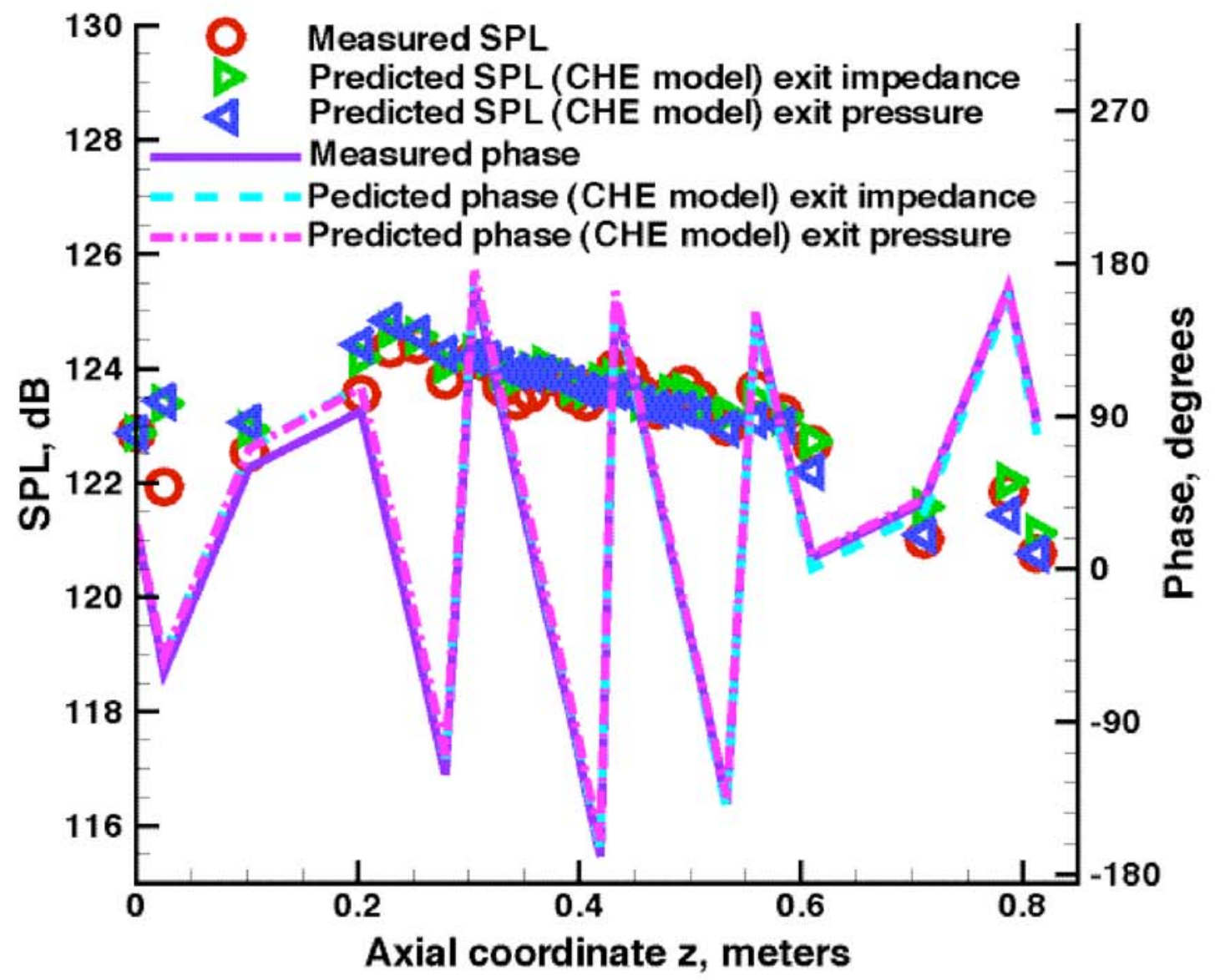

Fig 13b. Comparison of the measured lower wall sound pressure level (SPL) and phase at $3.0 \mathrm{kHz}$ with that produced by the CHE model using measured data, the measured exit impedance, the exit pressure boundary condition, and no flow: shows better comparison to measured data using the exit pressure boundary condition. 